\title{
Interlaminar Stress Analysis of Composite Shell Structures using a Geometrically Nonlinear Layer-wise Shell Finite Element
}

\author{
Zahra Soltani ${ }^{\mathrm{a} *}$, Seyed Ali Hosseini Kordkheili ${ }^{\mathrm{b}}$ \\ ${ }^{a}$ Department of Aeronautics, Imperial College London, London SW7 2AZ, UK \\ ${ }^{b}$ Center of Research and Development in Space Science and Technology, Aerospace \\ Engineering Department, Sharif University of Technology, Tehran, Iran
}

\begin{abstract}
This work aims to calculate interlaminar stress distribution through the thickness of multilayered composite shell structures by employing a novel nonlinear layer-wise shell finite element formulation. Adapting the Mindlin- Reissner theory in each layer, the shear-deformable layer-wise shell element presents the interlaminar shear stress distributions by increasing the number of layers. The interlaminar normal stress distribution is then determined by the finite difference solution of the general form of equilibrium equation in the non-orthogonal curvilinear grid along the Gaussian points. Two boundary conditions at the bottom and the top surfaces are satisfied by adopting the linear Lagrange interpolation function. The developed formulation is assessed through some illustrative problems solved using a proprietary finite element computer program. The results compare very well with those available in the literature and those obtained by simulations with the commercial finite element software Ansys.
\end{abstract}

Keywords: Composite Shell Structures, Interlaminar Stress Distributions, Layer-wise Shell Finite Element, Geometrically Nonlinear Analysis

\footnotetext{
* Corresponding author

Email address: Z.Soltani@imperial.ac.uk (Zahra Soltani ${ }^{\mathrm{a}}$ )
} 


\section{Introduction}

Composite shell structures provide improved structural benefits in many industrial applications by carrying loads mainly in direct compression or tension, rather than in bending or shear. In analysis of composite and sandwich lay-

5 ered shell structures, the first and higher-order shear-deformable theories are developed via adapting a continuous displacement, and subsequently continuous in-plane strain field along the shell thickness, which make them incapable of presenting the continuous distribution of interlaminar shear stress components. In addition, these theories assume a constant displacement in the shell-thickness direction and cannot take account of the interlaminar normal stresses. Owing to the fact that interlaminar strength is at least one order of magnitude smaller than that in fiber direction, the integrity of composite laminate shells is often challenged by delamination even when the interlaminar stresses are much smaller than those in the in-plane directions [1]. Furthermore, interlaminar normal stresses are needed to make the analysis capable of accounting for the shell thickness change, which is especially important in the analysis of sandwich shells with soft cores [2]. Numerous shell theories can be broadly classified into two categories, the equivalent single-layer (ESL) theories and the layer-wise theories. ESL theories, with low computational cost, provide a sufficiently accurate description of the global laminate response, except that they are often inadequate to determine 3D stress field at the ply level. In comparison with ESL theories, the layer-wise theories give a good representation of 3D stress fields by assuming separate displacement field expansions at each layer of laminated composites. Layer-wise shell models are used more and more with increasing use 25 of composite materials in moderately thick- and thick-walled curved structures, such as Air-intakes of formula race cars or strongly curved regions of ship hulls, where the interlaminar stresses play an important failure role.

In the context of numerical finite element methods, Kulikov and Plotnikova [3] presented a four-node curved shell element on the basis of a new nonlinear so strain-displacement relationship, written in local curvilinear coordinates, which 
are invariant under all rigid-body motions. By simultaneous solving of equilibrium equations for incremental displacements and assumed strains and stress resultants, their approach allows to use large load increments in nonlinear solution. Based on the co-rotational formulation, Marinković et al. [4] addressed the weaknesses of low-order interpolation shell finite elements in geometircally nonlinear analysis that also covers the electro-mechanical coupling. Rah et al. [5] introduced a constraint equation on the interlaminar stresses in the Fraeijs de Veubeke-Hu-Washizu (FHW) principle-based enhanced assumed strains formulation. A novel semi-analytic model is developed by González-Cantero et 40 al. [6] to evaluate non-regularized interlaminar stresses in laminates including highly curved parts, prone to unfolding failure. Nagle et al. [7] presented the interlaminar normal stress distribution across the width of L-shaped composite components in bending, with special emphasis on the free edge effects and the induced loading due to boundary conditions. Interlaminar stress in corru-

45 gated laminates studied by Thurnherr et al. [8]. Filipovic and Kress [9] studied the stress distribution in corrugated orthotropic laminates under shear loading. Numerical analysis of interlaminar stresses in open-hole laminates under compressive loads presented by Solis et al. [10]. Kulikov and Carrera [11] developed the higher-order shell models in a very compact form by employing the new concept of interpolation surfaces inside the shell body and in the framework of the Lagrangian description in the thickness direction. Proposing a new higherorder zigzag function, Carrera et al. [12] presented a new class of refined shell displacement-based theories that can provide a layer-wise kinematic description using equivalent-single-layer zigzag power functions. By making use of the node-variable plate theory assumptions, Valvano and Carrera [13] proposed a new plate finite element formulation for the analysis of composite and sandwich plates, which is capable for a simultaneous analysis of different subregions of the problem domain with different kinematics and accuracy. Filippi et al. [14] applied a number of higher-order plate elements and an improved theory based on a zig-zag power function to metallic and composite layered structures with viscoelastic layers. Implementing a variable-kinematic model, Pagani et al. [15] 
adopted refined approximations and layer-wise descriptions of the primary mechanical variables in selected portions of the layered structures that require a more accurate analysis. Nguyen et al. [16] proposed an efficient higher-order zig-zag theory to analyze the viscoelastic response of doubly-curved laminated shell structures. Kumar et al. [17] carried out a comprehensive free-vibration analysis on delaminated composite plates and shells for most of the theories using refined and advanced shell models contained in the Unified Formulation by Carrera (CUF) [18], in which the Mixed Interpolation of Tensorial Components (MITC) method is used to avoid shear locking and membrane locking. Studying delamination-prone free edge regions, Hajikazemi and Van Paepegem [19] developed a variational model to exactly determine both stress and displacement fields at free edges of general symmetric composite laminate strips under thermomechanical loads. Wowk et la. [20] discussed the effective method

75 for evaluating the edge-effect phenomenon early in the design process, using macroscopic finite element modeling.

In this paper the distribution of interlaminar shear stresses through the thickness of multilayered shell structures are calculated using a geometrically nonlinear layer-wise shell finite element formulation. The layer-wise shell finite element is a shear-deformable degenerated element with 8 nodes on the midsurface and $3+2 \mathrm{n}$ degrees of freedom per each node, in which $\mathrm{n}$ stands for the number of layers. By applying the Mindlin-Reissner theory in each layer, the present degenerated layer-wise shell element is able to calculate the distribution of interlaminar shear stresses through the thickness of a shell. Providing the correct distributions of interlaminar shear stresses, the interlaminar normal stress distribution is calculated using the general form of the equilibrium equation in the non-orthogonal curvilinear coordinate system of the layer-wise shell element. The 1st-order differential equation of equilibrium is solved by employing the finite-difference-method in a curvilinear grid along the Gaussian points.

90 Two boundary conditions of interlaminar normal stress at the bottom and the top surfaces are satisfied by adapting the linear Lagrange interpolation. The performance of the presented formulation is assessed by developing a propri- 
etary nonlinear finite element program and solving some illustrative problems. Results are compared with those available in the literature or obtained from the finite element (FE) analysis in Ansys.

\section{Interlaminar shear stress distributions using the layer-wise shell element}

The section starts with a brief description of the kinematics of the layer-wise shell element as a shear-deformable shell element. It proceeds to present the nonlinear finite-element formulation derived using a linearized form of the principle of virtual work. Finally, the distribution of interlaminar shear stresses along the thickness of composite shell structure is calculated using the layer-wise shell finite element.

\subsection{Kinematics of the geometrically nonlinear layer-wise shell element}

The layer-wise shell element is an eight-noded degenerated shell element that is formulated based on layer-wise defined rotation fields discontinuous across the layers. The nodes are placed on the mid-surface of the element and feature three translational plus $2 n$ rotational degrees of freedom per node, where $n$ stands for the number of layers. Adopting the Mindlin-Reissner theory in each layer, rotational degrees of freedom for layer $l$ are defined about two tangential orthogonal vectors ${ }^{t} \hat{\boldsymbol{V}}_{1 k}^{l}$ and ${ }^{t} \hat{\boldsymbol{V}}_{2 k}^{l}$ on the reference surface of the respective layer, with corresponding values of $\alpha_{k}^{l}$ and $\beta_{k}^{l}$, as shown schematically for a 3-layers element in Fig 1. It should be noted that in consistency with the continuity requirement between layers, the reference layer for mid, upper and lower layers are defined as the mid, lower and bottom surface, respectively. Using this kinematic hypothesis, the incremental displacement field in each layer $l$ is expressed as follows:

$$
\boldsymbol{u}^{l}(\xi, \eta, \zeta)=\sum_{k=1}^{8} N_{k}(\xi, \zeta)_{r e f} \boldsymbol{u}_{k}^{l}+\zeta^{l} \sum_{k=1}^{8} N_{k}(\xi, \zeta) t_{k}^{l} \hat{\boldsymbol{V}}_{3 k}^{l}
$$


where ${ }_{r e f} \boldsymbol{u}_{k}^{l}$ represents the incremental displacement field on the $l^{\text {th }}$ layer's reference surface. $\zeta^{l}$ is the layer thickness parameter measured with respect to the $l^{t h}$ layer's reference surface, as illustrated in Fig 1, and has the variation ranges of $[0,1],[-1,0]$ and $[-0.5,0.5]$ for upper, lower and mid layers, respectively. Also, $N_{k}(\xi, \zeta), a_{k}^{l}$ and $\hat{\boldsymbol{V}}_{3 k}^{l}$ are shape functions, thickness, and the increment in director vector of layer $l$ at $k^{\text {th }}$ node.

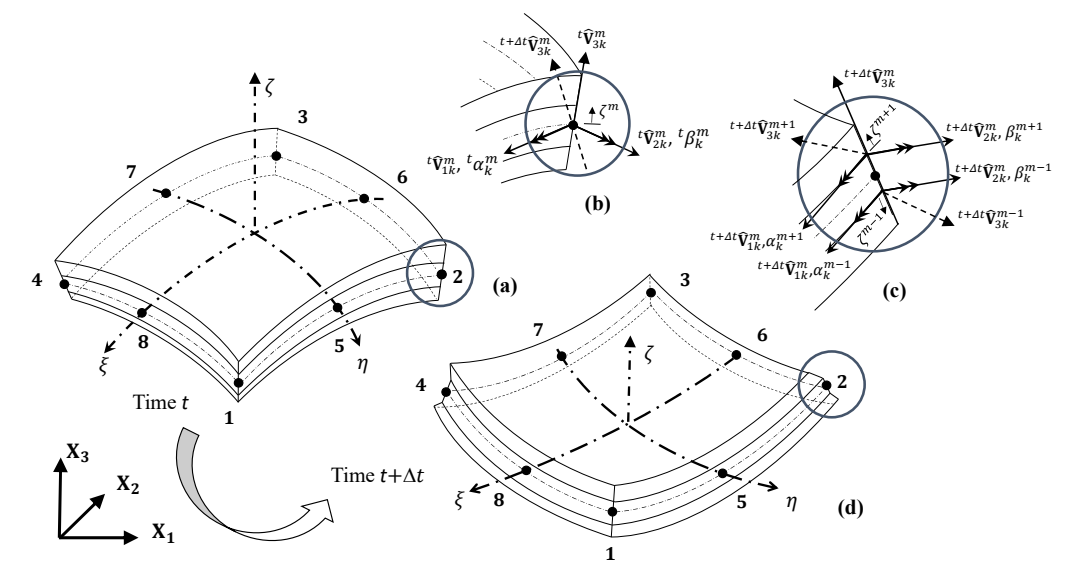

Figure 1: (a) Schematic of the layer-wise shell element with 3 layers at time $t$ (b) rotations of all layers around director vectors of mid-surface (c) extra ordered rotations for upper/lower layers about updated tangent vectors of mid-surface (d) configuration at time $t+\Delta t$.

The increment in the director vector of the mid layer $m$, with finite rotations of $\alpha_{k}^{m}$ and $\beta_{k}^{m}$ about two vectors $\hat{\boldsymbol{V}}_{1 k}^{m}$ and $\hat{\boldsymbol{V}}_{2 k}^{m}$, Fig $1(\mathrm{~b})$, is estimated using the second-order approximation scheme for finite rotation values [21] as follows:

$$
\hat{\boldsymbol{V}}_{3 k}^{m}={ }^{t+\Delta t} \hat{\boldsymbol{V}}_{3 k}^{m}-{ }^{t} \hat{\boldsymbol{V}}_{3 k}^{m}={ }^{t} \boldsymbol{g}_{1 k}^{m} \alpha_{k}^{m}+{ }^{t} \boldsymbol{g}_{2 k}^{m} \beta_{k}^{m}
$$

where

$$
\begin{aligned}
{ }^{t} \boldsymbol{g}_{1 k}^{m} & =-\left({ }^{t} \hat{\boldsymbol{V}}_{2 k}^{m}+0.5 \alpha_{k}^{m t} \hat{\boldsymbol{V}}_{3 k}^{m}\right) \\
{ }^{t} \boldsymbol{g}_{2 k}^{m} & ={ }^{t} \hat{\boldsymbol{V}}_{1 k}^{m}+0.5 \beta_{k}^{m t} \hat{\boldsymbol{V}}_{3 k}^{m}
\end{aligned}
$$


The increment in the director vector of upper/lower layer $l$ in Fig 1 can be obtained by adding the increment in director vector of the mid layer, Fig 1(b), and the increment due to extra rotations $\alpha_{k}^{l}$ and $\beta_{k}^{l}$ that are defined about the updated tangential vectors of the mid layer ${ }^{t+\Delta t} \hat{\boldsymbol{V}}_{1 k}^{m}$ and ${ }^{t+\Delta t} \hat{\boldsymbol{V}}_{2 k}^{m}$, Fig 1(c). In the general case, for a layer-wise shell element with $n$ layers, the ordered algorithm includes the vector addition of individual increments due to successive rotations $\alpha_{k}^{j}$ and $\beta_{k}^{j}$ about the updated tangential vectors of respective previous 135 layers from the mid layer till the intended layer. In this way and by adapting the second-order approximation Eq (2) for calculation of individual increments, the increment in the director vector of upper/lower layer $l$ can be expressed as:

$$
\hat{\boldsymbol{V}}_{3 k}^{l}= \begin{cases}\hat{\boldsymbol{V}}_{3 k}^{m}+\sum_{j=m+1}^{l}\left({ }^{t} \boldsymbol{g}_{1 k}^{j}{ }^{t} \alpha_{k}^{j}+{ }^{t} \boldsymbol{g}_{2 k}^{j}{ }^{t} \beta_{k}^{j}\right) & l=1, \ldots, m-1 \\ \hat{\boldsymbol{V}}_{3 k}^{m}+\sum_{j=l}^{m-1}\left({ }^{t} \boldsymbol{g}_{1 k}^{j}{ }^{t} \alpha_{k}^{j}+{ }^{t} \boldsymbol{g}_{2 k}^{j}{ }^{t} \beta_{k}^{j}\right) & l=m+1, \ldots, n\end{cases}
$$

where

$$
\begin{gathered}
{ }^{t} \hat{\boldsymbol{g}}_{1 k}^{l}= \begin{cases}-\left({ }^{t+\Delta t} \hat{\boldsymbol{V}}_{2 k}^{l+1}+0.5 \alpha_{k}^{l}{ }^{t+\Delta t} \boldsymbol{V}_{1 k}^{l+1}\right) & l=1, \ldots, m-1 \\
-\left({ }^{t+\Delta t} \hat{\boldsymbol{V}}_{2 k}^{l-1}+0.5 \alpha_{k}^{l}{ }^{t+\Delta t} \boldsymbol{V}_{3 k}^{l-1}\right) & l=1, \ldots, m-1\end{cases} \\
{ }^{t} \hat{\boldsymbol{g}}_{2 k}^{l}= \begin{cases}{ }^{t+\Delta t} \hat{\boldsymbol{V}}_{1 k}^{l+1}-0.5 \beta_{k}^{l t+\Delta t} \boldsymbol{V}_{1 k}^{l+1} & l=1, \ldots, m-1 \\
{ }^{t+\Delta t} \hat{\boldsymbol{V}}_{1 k}^{l-1}-0.5 \beta_{k}^{l}{ }^{t+\Delta t} \boldsymbol{V}_{3 k}^{l-1} & l=1, \ldots, m-1\end{cases}
\end{gathered}
$$

The incremental displacement field on the $l^{\text {th }}$ layer's reference surface, ref $\boldsymbol{u}_{k}^{l}$ in Eq (1), is obtained by adding the nodal displacement values $\boldsymbol{u}_{k}$ and those caused by finite rotations of previous layers from the mid layer till the reference 
surface of layer $l$ as follows:

$$
r e f^{\boldsymbol{u}_{k}^{l}}= \begin{cases}\boldsymbol{u}_{k}-\left(t_{k}^{m} / 2+\sum_{j=l+1}^{m-1} t_{k}^{j}\right) \hat{\boldsymbol{V}}_{3 k}^{m}-\sum_{i=l+1}^{m-1} \sum_{j=l+1}^{i} t_{k}^{j} \Delta \boldsymbol{V}_{k}^{i} & l=1, \ldots, m-1 \\ \boldsymbol{u}_{k} & l=m \\ \boldsymbol{u}_{k}-\left(t_{k}^{m} / 2+\sum_{j=m+1}^{l-1} t_{k}^{j}\right) \hat{\boldsymbol{V}}_{3 k}^{m}-\sum_{i=m+1}^{l-1} \sum_{j=i}^{l-1} t_{k}^{j} \Delta \boldsymbol{V}_{k}^{i} & l=m+1, \ldots, n\end{cases}
$$

where

$$
\Delta \boldsymbol{V}_{k}^{l}={ }^{t} \boldsymbol{g}_{1 k t}^{l} \alpha_{k}^{l}+{ }^{t} \boldsymbol{g}_{2 k t}^{l} \beta_{k}^{l}
$$

The equilibrium of the layer-wise shell element at time $t+\Delta t$ is expressed using the principle of virtual displacements in the Updated Lagrangian (UL) framework and taking the summation over individual layers as follows:

$$
\int_{{ }^{t} V}{ }^{t+\Delta t} S_{i j} \delta_{t}^{t+\Delta t} \epsilon_{i j} d^{t} V=\sum_{l=1}^{n} \int_{{ }^{t} V^{j}}{ }^{t+\Delta t} S_{i j}^{l} \delta_{t}^{t+\Delta t} \epsilon_{i j}^{l} d^{t} V^{l}={ }^{t+\Delta t} \mathfrak{R}
$$

where ${ }_{t}^{t+\Delta t} S_{i j}^{l}$ and ${ }_{t}^{t+\Delta t} \epsilon_{i j}^{l}$ are the 2nd-Piola Kirchhoff (PK2) stress and Green 145 virtual work. The PK2 stress GL strain tensors form the energy conjugate pair for geometrically nonlinear analysis of structures with large displacements, large rotations but small strains. The pair can be used in the Total Lagrangian (TL) as well as the Updated Lagrangian (UL) frameworks. The present formulation is developed based on the UL incremental approach, which has more numerical effectiveness in comparison with the TL formulation [22].

In order to be able to calculate integral terms with different ranges of $\zeta^{l}$ appearing in the finite element formulation, the components of the GL strain tensor are expanded as second-order functions in terms of the thickness param- 


$$
{ }_{t}^{t+\Delta t} \epsilon_{i j}^{l}={ }_{t} \epsilon_{i j}^{l}=\frac{1}{2}\left(u_{i, j}^{l}+u_{j, i}^{l}+u_{k, i}^{l} u_{k, j}^{l}\right)={ }_{t} \epsilon_{i j}^{l(0)}+\zeta_{t}^{l} \epsilon_{i j}^{l(1)}+\zeta^{l^{2}}{ }_{t} \epsilon_{i j}^{l(2)}
$$

where

$$
\begin{aligned}
{ }_{t} \epsilon_{i j}^{l(0)} & =\frac{1}{2} \sum_{k=1}^{8}\left[\left(N_{k, j \text { ref }} \boldsymbol{u}_{k}^{l}(i)+N_{k, i \text { ref }} \boldsymbol{u}_{k}^{l}(j)\right)+N_{k} t_{k}^{l}\left(J_{j 3}^{-1} \hat{\boldsymbol{V}}_{3 k}^{m}(i)\right.\right. \\
& \left.+J_{i 3}^{-1} \hat{\boldsymbol{V}}_{3 k}^{m}(j)\right) \sum_{k^{\prime}=1}^{8}\left(N_{k, i r e f} \boldsymbol{u}_{k}^{l}(p)+N_{k} t_{k}^{l} J_{i 3}^{-1} \hat{\boldsymbol{V}}_{3 k}^{m}(p)\right) \\
& \left.\left(N_{k^{\prime}, j \operatorname{rref}} \boldsymbol{u}_{k^{\prime}}^{l}(p)+N_{k^{\prime}} t_{k^{\prime}}^{l}, J_{j 3}^{-1} \hat{\boldsymbol{V}}_{3 k^{\prime}}^{m}(p)\right)\right] \\
{ }_{t} \epsilon_{i j}^{l(1)}= & \frac{1}{2} \sum_{k=1}^{8}\left[t_{k}^{l}\left(N_{k, j} \hat{\boldsymbol{V}}_{3 k}^{m}(i)+N_{k, i} \hat{\boldsymbol{V}}_{3 k}^{m}(j)\right)+\sum_{k^{\prime}=1}^{8}\left[\left(N_{k, i r e f} \boldsymbol{u}_{k}^{l}(p)\right.\right.\right. \\
+ & \left.N_{k} t_{k}^{l} J_{i 3}^{-1} \hat{\boldsymbol{V}}_{3 k}^{m}(p)\right) N_{k^{\prime}, j} t_{k^{\prime}}^{l} \hat{\boldsymbol{V}}_{3 k^{\prime}}^{m}(p)+\left(N_{k^{\prime}, j} r e f \boldsymbol{u}_{k^{\prime}}^{l}(p)\right. \\
+ & \left.\left.\left.N_{k^{\prime}} t_{k^{\prime}}^{l} J_{j 3}^{-1} \hat{\boldsymbol{V}}_{3 k^{\prime}}^{m}(p)\right) N_{k^{\prime}, i} t_{k}^{l} \hat{\boldsymbol{V}}_{3 k^{\prime}}^{m}(p)\right]\right] \\
& { }_{t} \epsilon_{i j}^{l(2)}=\frac{1}{2} \sum_{k=1}^{8} \sum_{k^{\prime}=1}^{8} a_{k}^{l} a_{k^{\prime}}^{l} N_{k, j} N_{k^{\prime}, i} \hat{\boldsymbol{V}}_{3 k}^{m}(p) \hat{\boldsymbol{V}}_{3 k^{\prime}}^{m}(p)
\end{aligned}
$$

where the left subscript $t$ denotes the incremental value. Substituting Eq (9) into the incremental stress-strain relationship, ${ }_{t} S_{i j}^{l}={ }_{t} C_{i j r s}^{l} \epsilon_{r s}^{l}$, the incremental PK2 tensor and then Cauchy stress tensor can be also rewritten as follows: where

$$
\begin{gathered}
{ }_{t} S_{i j}^{l}={ }_{t} S_{i j}^{l(0)}+\zeta^{l}{ }_{t} S_{i j}^{l(1)}+\zeta^{l^{2}}{ }_{t} S_{i j}^{l(2)} \\
{ }^{t+\Delta t} \sigma_{i j}^{l}{ }^{t+\Delta t} \sigma_{i j}^{l(0)}+\zeta^{l t+\Delta t} \sigma_{i j}^{l(1)}+\zeta^{l^{2} t+\Delta t} \sigma_{i j}^{l(2)}
\end{gathered}
$$

where 


$$
\begin{gathered}
{ }_{t} S_{i j}^{l(\psi)}={ }_{t} C_{i j r s t}^{l} \epsilon_{i j}^{l(\psi)} \\
{ }_{t} \sigma_{i j}^{l(\psi)}={ }_{t}^{t+\Delta t} x_{m, i}{ }_{t}^{t+\Delta t} S_{i j}^{l(\psi)}{ }_{t}^{t+\Delta t} x_{n, i} / \operatorname{det}\left({ }_{t}^{t+\Delta t} \boldsymbol{X}\right)
\end{gathered}
$$

where ${ }_{t}^{t+\Delta t} \boldsymbol{X}=\left[{ }_{t}^{t+\Delta t} x_{i, j}\right]=\left[\partial^{t+\Delta t} x_{i} / \partial^{t} x_{j}\right]$ denotes the deformation gradient tensor.

Now, substituting Eqs (13) and (14) in the incremental decomposed form of the PK2 stress tensor, ${ }_{t}^{t+\Delta t} S_{i j}^{l}={ }^{t} \sigma_{i j}^{l}+{ }_{t} S_{i j}^{l}$, results in following expansion of ${ }_{t}^{t+\Delta t} S_{i j}$ in terms of $\zeta^{l}$

$$
{ }_{t}^{t+\Delta t} S_{i j}^{l}=\left({ }^{t} \sigma_{i j}^{l(0)}+{ }_{t} S_{i j}^{l(0)}\right)+\zeta^{l}\left({ }^{t} \sigma_{i j}^{l(1)}+{ }_{t} S_{i j}^{l(1)}\right)+\zeta^{l^{2}}\left({ }^{t} \sigma_{i j}^{l(2)}+{ }_{t} S_{i j}^{l(2)}\right)
$$

Finally, substituting Eq (17) into Eq (8) and employing the approximation 165

relation ${ }_{t} S_{i j}^{l(0)}={ }_{t} C_{i j r s t}^{l} e_{r s}^{l(0)}$, the linearized form of the equilibrium equation at time $t+\Delta t$ is obtained after some mathematical manipulation as follows:

$$
\begin{aligned}
& \sum_{l=1}^{n} \int_{{ }^{t} V^{l}}{ }^{t} C_{i j r s}^{l}{ }^{t} e_{r s}^{l(0)} \delta^{t} e_{i j}^{l} d^{t} V^{l}+\sum_{l=1}^{n} \int_{{ }^{t} V^{l}}\left({ }_{t} S_{i j}^{l(0)}+\zeta^{l}{ }_{t} S_{i j}^{l(1)}+\zeta^{{ }^{2}}{ }_{t} S_{i j}^{l(2)}\right) \delta^{t} \eta_{i j}^{l} d^{t} V^{l} \\
& ={ }^{t+\Delta t} \mathfrak{R}-\sum_{l=1}^{n} \int_{{ }^{t} V^{l}}\left({ }^{t+\Delta t} \sigma_{i j}^{l(0)}+\zeta_{t}^{l+\Delta t}{ }^{t+\Delta t} S_{i j}^{l(1)}+\zeta^{l^{2}}{ }_{t}^{t+\Delta t} S_{i j}^{l(2)}\right) \delta^{t} e_{i j}^{l} d^{t} V^{l}
\end{aligned}
$$

where ${ }_{t} e_{i j}^{l}=\frac{1}{2}\left(u_{i, j}^{l}+u_{j, i}^{l}\right)$ and ${ }_{t} \eta_{i j}^{l}=\frac{1}{2} u_{k, i}^{l} u_{k, j}^{l}$ are the linear and nonlinear parts of the incremental GL strain tensor. In turn, the linear and nonlinear strain-displacement transformation matrices $\boldsymbol{B}_{L}^{l}$ and $\boldsymbol{B}_{N L}^{l}$ can be expanded in 170 terms of $\zeta^{l}$ as follows:

$$
\begin{gathered}
{ }_{t}^{t+\Delta t} \boldsymbol{B}_{L}^{l}={ }_{t}^{t+\Delta t} \boldsymbol{B}_{L a}^{l}+\zeta_{t}^{l+\Delta t} \boldsymbol{B}_{L b}^{l} \\
{ }_{t}^{t+\Delta t} \boldsymbol{B}_{N L}^{l}={ }_{t}^{t+\Delta t} \boldsymbol{B}_{N L a}^{l}+\zeta^{l}{ }_{t}^{t+\Delta t} \boldsymbol{B}_{N L b}^{l}
\end{gathered}
$$


Now, employing the explicit integration method along the thickness direction, the incremental finite element form of the equilibrium equation at $i^{\text {th }}$ Newton-Raphson iteration will be as follows:

$$
\begin{gathered}
\left({ }_{t}^{t+\Delta t} \boldsymbol{K}_{L}^{(i-1)}+{ }_{t}^{t+\Delta t} \boldsymbol{K}_{N L}^{(i-1)}\right) \Delta \boldsymbol{U}^{(i)}={ }^{t+\Delta t} \mathfrak{R}-{ }_{t}^{t+\Delta t} \boldsymbol{F}^{(i-1)} \\
{ }_{t}^{t+\Delta t} \boldsymbol{U}^{(i)}={ }_{t}^{t+\Delta t} \boldsymbol{U}^{(i-1)}+\Delta \boldsymbol{U}^{(i)}
\end{gathered}
$$

where ${ }_{t}^{t+\Delta t} \boldsymbol{K}_{L}^{(i-1)},{ }_{t}^{t+\Delta t} \boldsymbol{K}_{N L}^{(i-1)}$ and ${ }_{t}^{t+\Delta t} \boldsymbol{F}^{(i-1)}$ are, respectively, the linear and nonlinear stiffness matrices and the internal force vector at the $(i-1)^{t h}$ iteration which are calculated by taking summation over all layers as follows:

$$
\begin{gathered}
{ }_{t}^{t+\Delta t} \boldsymbol{K}_{L}=\left.\left(\sum_{l=1}^{m-1}{ }_{t}^{t+\Delta t} \boldsymbol{K}_{L \downarrow}^{l}+{ }_{t}^{t+\Delta t} \boldsymbol{K}_{L}^{m}+\sum_{l=m+1}^{n}{ }_{t}^{t+\Delta t} \boldsymbol{K}_{L \uparrow}^{l}\right)\right|^{t} \boldsymbol{J}_{(\xi, \eta, \zeta=0)} \mid \\
{ }_{t}^{t+\Delta t} \boldsymbol{K}_{N L}=\left.\left(\sum_{l=1}^{m-1}{ }_{t}^{t+\Delta t} \boldsymbol{K}_{N L \downarrow}^{l}+{ }_{t}^{t+\Delta t} \boldsymbol{K}_{N L}^{m}+\sum_{l=m+1}^{n}{ }_{t}^{t+\Delta t} \boldsymbol{K}_{N L \uparrow}^{l}\right)\right|^{t} \boldsymbol{J}_{(\xi, \eta, \zeta=0)} \mid \\
{ }_{t}^{t+\Delta t} \boldsymbol{F}=\left(\sum_{l=1}^{m-1}{ }^{t+\Delta t} \boldsymbol{F}_{\downarrow}^{l}+{ }_{t}^{t+\Delta t} \boldsymbol{F}^{m}+\sum_{l=m+1}^{n}{ }_{t}^{t+\Delta t} \boldsymbol{F}_{\uparrow}^{l}\right)\left|{ }^{t} \boldsymbol{J}_{(\xi, \eta, \zeta=0)}\right|
\end{gathered}
$$

where, for the seek of simplicity, the superscript $(i-1)$ is dropped. Also, ${ }_{t}^{t+\Delta t} \boldsymbol{K}_{L \downarrow}^{l},{ }_{t}^{t+\Delta t} \boldsymbol{K}_{N L \downarrow}^{l}$ and ${ }_{t}^{t+\Delta t} \boldsymbol{F}_{\downarrow}^{l}$ denotes linear, nonlinear stiffness matrices and internal load vector for lower layers and ${ }_{t}^{t+\Delta t} \boldsymbol{K}_{L \uparrow}^{l},{ }_{t}^{t+\Delta t} \boldsymbol{K}_{N L \uparrow}^{l}$ and ${ }_{t}^{t+\Delta t} \boldsymbol{F}_{\uparrow}^{l}$ are those for upper layers which are defined in Appendix A. In the above equations, $\boldsymbol{J}_{(\xi, \eta, \zeta=0)}$ is determinant of Jacobian matrix on the mid-surface of the shell element.

\subsection{Interlaminar Shear Stress Distributions}

Interlaminar shear-stress components must be continuous on the interfaces of layers and be in equilibrium with the applied values on boundaries. The layerwise shell element employs the Mindlin-Reissner theory in each layer which 
implies constant values of interlaminar shear stresses through the thickness of each layer. Depending on the curvature of the shell structure, interlaminar shear stress components at each Gaussian point are expressed in the local orthogonal coordinate system using the following transformation:

$$
{ }^{t+\Delta t} \boldsymbol{\sigma}^{\prime l}={ }^{t+\Delta t} \boldsymbol{L}^{T t+\Delta t} \boldsymbol{\sigma}^{l t+\Delta t} \boldsymbol{L}
$$

where

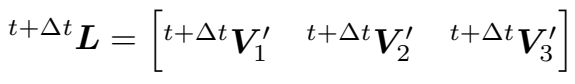

and ${ }^{t+\Delta t} \boldsymbol{V}_{1}^{\prime}$ and ${ }^{t+\Delta t} \boldsymbol{V}_{2}^{\prime}$ are unit orthogonal vectors on the element mid-surface at Gaussian points $G P$, and ${ }^{t+\Delta t} \boldsymbol{V}_{3}^{\prime}$ is the unit normal vector along the thickness direction, as it is shown in Fig 2(a) where for simplicity the left superscript $(t+\Delta t)$ is dropped.

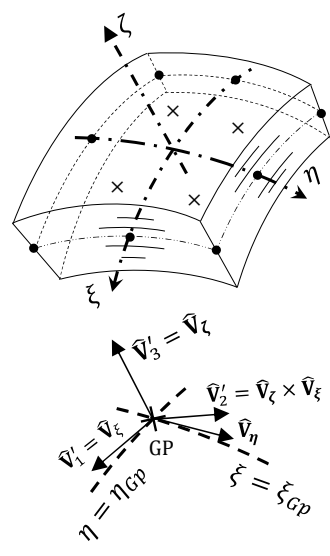

(a)

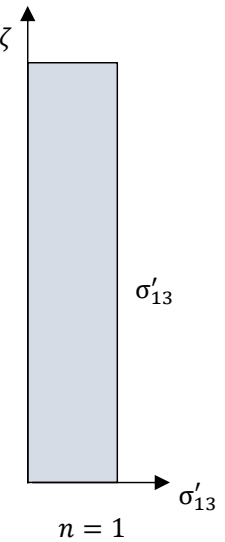

(b)

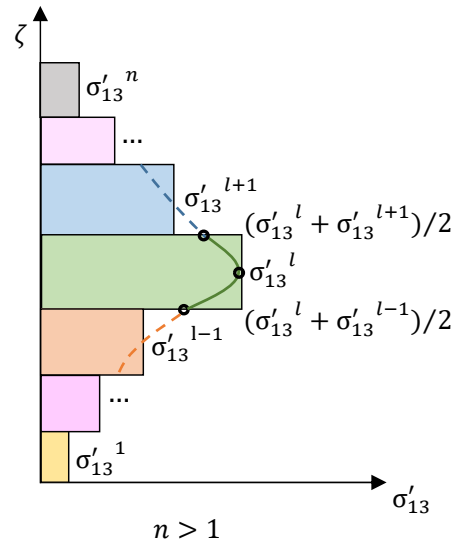

(c)

Figure 2: (a) The local curvilinear and orthogonal coordinate systems at each Gaussian point. Interlaminar shear-stress distribution in the local orthogonal coordinate system using the element with the number of layers (b) $n=1$ and (c) $n>1$.

With increasing number of computational layers in this shear-deformable element, the difference between shear stress values at adjacent layers decreases, as it is shown schematically in Fig 2(b) to (c). The number of layers in layerwise shell element will be increased until the piece-wise constant distribution 
of shear stress (Fig 2(c)) meets the convergence criterion. Then, the continuity condition will be applied by assigning the average value of shear stress at two adjacent layers of each interface. Finally, the continuous distributions of interlaminar shear stress will be obtained by employing the Lagrange second-order interpolation polynomials in each layer $l$ as follows:

$$
\sigma_{i 3}^{\prime l}(\xi, \eta, \zeta)=\frac{P_{l-1}}{2} \sigma_{i 3}^{\prime l-1}+\frac{P_{l-1}+2 P_{l}+P_{l+1}}{2} \sigma_{i 3}^{\prime l}+\frac{P_{l+1}}{2} \sigma_{i 3}^{\prime l+1} \quad i=1,2
$$

where $P_{l-1}(\xi, \eta, \zeta), P_{l}(\xi, \eta, \zeta)$ and $P_{l+1}(\xi, \eta, \zeta)$ are the Lagrange second-order basis polynomials at, respectively, the bottom, middle and top of the layer with following expressions:

$$
\begin{gathered}
P_{l-1}(\xi, \eta, \zeta)=\frac{1}{2\left(t^{l}\right)^{2}}\left(t(1+\zeta)-2 \mathbb{t}-2 t^{l}\right)\left(t(1+\zeta)-2 \mathbb{t}-t^{l}\right) \\
P_{l}(\xi, \eta, \zeta)=\frac{1}{\left(t^{l}\right)^{2}}(2 \mathbb{t}-t(1+\zeta))\left(t(1+\zeta)-2 \mathbb{t}-2 t^{l}\right) \\
P_{l+1}(\xi, \eta, \zeta)=\frac{1}{2\left(t^{l}\right)^{2}}(t(1+\zeta)-2 \mathbb{E})\left(t(1+\zeta)-2 \mathbb{t}-t^{l}\right)
\end{gathered}
$$

195 condition values should be applied.

\section{Interlaminar normal stress distribution}

Employing the typical assumption of the constant displacement in the thickness direction of shell structures, the layer-wise shell-element formulation is 
mid-surface in addition with rotational degrees of freedom about orthogonal directions normal to the director vectors, Eq (1). Derived based on the planestress theory, the formulation cannot take into account the interlaminar normal stresses and if interlaminar normal strains are calculated, they are determined from the membrane strains based on the Poisson effect [2]. In this section, the distribution of interlaminar normal stress is determined as a post-processing analysis and by using the differential equation of equilibrium along the thickness direction in the element curvilinear coordinate system.

The differential equations of equilibrium in a general non-orthogonal coordinate system have the following form:

$$
\sigma^{\beta \alpha} \|_{\beta}+\rho b^{\alpha}=\rho a^{\alpha} \quad \alpha, \beta=1,2,3
$$

where $\sigma^{\beta \alpha}, b^{\alpha}$ and $a^{\alpha}$ denote the contravariant stress tensor, body force and acceleration vectors, $\rho$ denotes mass density and the symbol $\|$ denotes contravariant differentiation [17]. A superscript symbol indicates that the variables are expressed in the element curvilinear coordinate system $x^{\beta}(\beta=1,2,3)$, rather than global system $x_{\beta}$. In the absence of body forces, the static form of the differential equilibrium equation (31) along the thickness direction $x^{3}$ of the layer-wise shell element reduces to:

$$
\frac{\partial \sigma^{\beta 3}}{\partial x^{\beta}}+\sigma^{\beta \gamma} \Gamma_{\beta \gamma}^{3}+\sigma^{\beta 3} \Gamma_{\beta \gamma}^{\gamma}=0 \quad \alpha, \beta=1,2,3
$$

where $\Gamma_{\beta \gamma}^{\alpha}$ denote Christoffel symbols of the second kind that show the variation of the basis vectors with position and will be calculated shortly afterwards. The first-order differential equation (32) will be solved by employing the finitedifference-method.

\subsection{Contravariant Stress Components and Christoffel symbols}

Contravariant stress components in the element curvilinear coordinate system are related to the stress tensor in the Cartesian coordinate system, Eq (14), by 
following transformation:

$$
\sigma^{\alpha \beta}=g^{\alpha \gamma} g^{\beta \delta} \sigma_{\gamma \delta}
$$

where $g^{\alpha \beta}$ is the contravariant metric tensor defined as:

$$
g^{\alpha \beta}=\frac{\partial x^{\alpha}}{\partial x^{\gamma}} \frac{\partial x^{\beta}}{\partial x^{\gamma}}
$$

220 tensor that defines the length of and angle between tangent vectors at each point:

$$
g^{\alpha \beta}=\frac{\partial x^{\gamma}}{\partial x^{\alpha}} \frac{\partial x^{\gamma}}{\partial x^{\beta}}=\hat{\boldsymbol{V}}_{\alpha} \cdot \hat{\boldsymbol{V}}_{\beta}
$$

where $\hat{\boldsymbol{V}}_{\xi}, \hat{\boldsymbol{V}}_{\eta}$ and $\hat{\boldsymbol{V}}_{\zeta}$ are shown in Fig 2(a).

Christoffel symbols of the second kind, $\Gamma_{\alpha \beta}^{\gamma}$, are defined as follows:

$$
\Gamma_{\alpha \beta}^{\gamma}=g^{\gamma \delta} \Gamma_{\alpha \beta \delta}
$$

225

where $\Gamma_{\alpha \beta \delta}$ are Christoffel symbols of the first kind that are defined, in turn by, following formula

$$
\Gamma_{\alpha \beta \gamma}=\frac{1}{2}\left(\frac{\partial g_{\alpha \gamma}}{\partial x^{\beta}}+\frac{\partial g_{\beta \gamma}}{\partial x^{\alpha}}-\frac{\partial g_{\alpha \beta}}{\partial x^{\gamma}}\right)=\frac{\partial^{2} x_{r}}{\partial x^{\alpha} \partial x^{\beta}} \frac{\partial x_{r}}{\partial x^{\gamma}}
$$

The geometry of the shell element with respect to the mid-surface is expressed as:

$$
\boldsymbol{X}(\xi, \eta, \zeta)=\sum_{k=1}^{8} N_{k}(\xi, \eta) \boldsymbol{x}_{k}+\frac{\zeta}{2} \sum_{k=1}^{8} N_{k}(\xi, \eta) t_{k}{ }^{t} \hat{\boldsymbol{V}}_{3 k}
$$

where $\boldsymbol{x}_{k}, t_{k}$ and ${ }^{t} \hat{\boldsymbol{V}}_{3 k}$ represent, respectively, the location, thickness and unit 230 vector normal to the mid-surface at $k^{\text {th }}$ node. Substituting Eq (38) in Eq (37), Christoffel symbols of the second kind will be obtained using the following formulae for $\alpha, \beta, \gamma=1,2$ : 


$$
\begin{gathered}
\Gamma_{\alpha \beta \gamma}=\left(\sum_{k=1}^{8} N_{k, \alpha \beta}{ }^{t} \boldsymbol{x}_{k}+\frac{\zeta}{2} \sum_{k=1}^{8} N_{k, \alpha \beta} t_{k}{ }^{t} \hat{\boldsymbol{V}}_{3 k}\right) \cdot\left(\sum_{k=1}^{8} N_{k, \gamma}{ }^{t} \boldsymbol{x}_{k}+\frac{\zeta}{2} \sum_{k=1}^{8} N_{k, \gamma} t_{k}{ }^{t} \hat{\boldsymbol{V}}_{3 k}\right) \\
\Gamma_{3 \alpha \beta}=\Gamma_{\alpha 3 \beta}=\frac{1}{2}\left(\sum_{k=1}^{8} N_{k, \alpha} t_{k}{ }^{t} \hat{\boldsymbol{V}}_{3 k}\right) \cdot\left(\sum_{k=1}^{8} N_{k, \beta}{ }^{t} \boldsymbol{x}_{k}+\frac{\zeta}{2} \sum_{k=1}^{8} N_{k, \beta} t_{k}{ }^{t} \hat{\boldsymbol{V}}_{3 k}\right) \\
\Gamma_{\alpha \beta 3}=\frac{1}{2}\left(\sum_{k=1}^{8} N_{k, \alpha \beta}{ }^{t} \boldsymbol{x}_{k}+\frac{\zeta}{2} \sum_{k=1}^{8} N_{k, \alpha \beta} t_{k}{ }^{t} \hat{\boldsymbol{V}}_{3 k}\right) \cdot\left(\sum_{k=1}^{8} N_{k} t_{k}{ }^{t} \hat{\boldsymbol{V}}_{3 k}\right) \\
\Gamma_{\alpha 33}=\Gamma_{3 \alpha 3}=\frac{1}{4}\left(\sum_{k=1}^{8} N_{k, \alpha} t_{k}{ }^{t} \hat{\boldsymbol{V}}_{3 k}\right) \cdot\left(\sum_{k=1}^{8} N_{k} t_{k}{ }^{t} \hat{\boldsymbol{V}}_{3 k}\right) \\
\Gamma_{33 \alpha}=0 .
\end{gathered}
$$

\subsection{Equilibrium differential equation finite-difference solution with two bound-} ary conditions

Considering $n p$ points through the thickness of shell element with the distance of $h_{\zeta}=t_{G P} /\left(n_{p}-1\right)$, Fig $3(\mathrm{a})$, interlaminar normal stress distribution at each Gauss point can be determined using finite-difference form of Eq (32) as follows:

$$
\begin{aligned}
\left(\frac{A}{h_{\zeta}}+2 \Gamma_{33}^{3}+\Gamma_{31}^{1}+\Gamma_{32}^{2}\right) \sigma_{\zeta}^{33} & =\frac{A}{h_{\zeta}} \sigma_{\zeta-A h_{\zeta}}^{33}-\sigma_{, 1}^{13}-\sigma_{, 2}^{23} \\
& -\left(\sigma^{\beta \gamma} \Gamma_{\beta \gamma}^{3}\right)_{(\beta, \gamma) \neq(3,3)}-\left(\sigma^{\beta 3} \Gamma_{\beta \gamma}^{\gamma}\right)_{\beta \neq 3}
\end{aligned}
$$

where $A=1$ and $A=-1$ is related to, respectively, the backward and forward finite-difference formula along the thickness direction. To satisfy two boundary conditions at the bottom and the top surfaces, the first order differential equation Eq (32) will be solved once using the boundary value at the bottom surface and the backward formula, $\sigma_{\uparrow}^{33}$, and once again using the forward formula and the top boundary value, $\sigma_{\downarrow}^{33}$. Then the correct value of interlaminar normal stress at each point is determined according to the distance from each boundary and using the linear Lagrange shape function: 


$$
\sigma^{33}=\frac{(1-\zeta)}{2} \sigma_{\uparrow}^{33}+\frac{(1+\zeta)}{2} \sigma_{\uparrow}^{33}
$$

\subsection{Curvilinear grid along Gaussian points}

To calculate derivatives of shear-stress components along the parametric lines $\xi$ and $\eta$ on the right hand side of Eq (44), one needs to calculate the distances between Gaussian points. Fig 3(b) shows the general non-orthogonal curvilinear
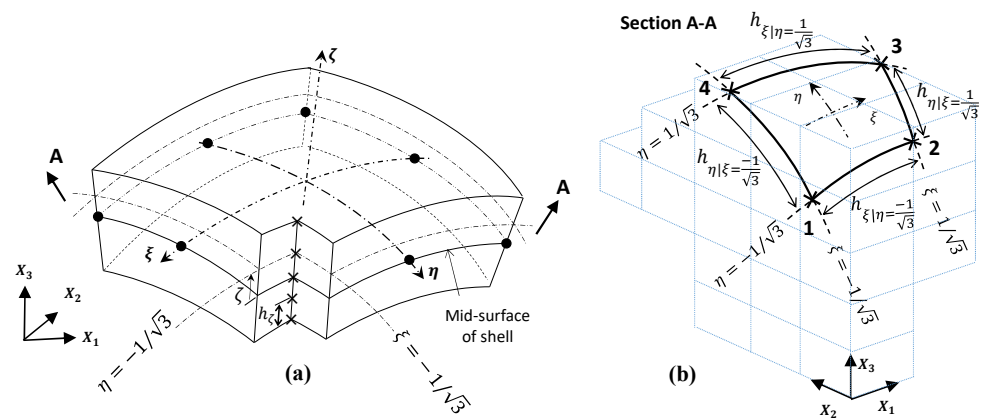

Figure 3: Non-orthogonal curvilinear grid along the Gaussian points at a typical section A-A of shell body at thickness coordinate of $\zeta$.

The differential length elements in the space defined by the geometry of shell, Eq (38), are expressed as follows:

$$
d \boldsymbol{X}=\boldsymbol{X}_{, \xi} d \xi+\boldsymbol{X}_{, \eta} d \eta+\boldsymbol{X}_{, \zeta} d \zeta
$$

Then the distances $h_{\xi}$ and $h_{\eta}$ along the parametric curves at distance of $\zeta$, Fig

$3(\mathrm{~b})$, can be calculated for $d \zeta=0$ and by setting $d \eta=0$ and $d \xi=0$, respectively. The values of integrals can be determined using the change of variable $\zeta^{\prime}=\sqrt{3} \zeta$ and $\eta^{\prime}=\sqrt{3} \eta$, respectively:

$$
h_{\left.\xi\right|_{\eta=\eta_{G P}}}=\left.\int_{-1 / \sqrt{3}}^{1 / \sqrt{3}} \sqrt{\boldsymbol{X}_{, \xi} \cdot \boldsymbol{X}_{, \xi}}\right|_{\eta=\eta_{G P}} d \xi=\left.\frac{1}{\sqrt{3}} \sum_{\xi= \pm \frac{1}{3}} \sqrt{\boldsymbol{X}_{, \xi} \cdot \boldsymbol{X}_{, \xi}}\right|_{\eta=\eta_{G P}}
$$




$$
h_{\left.\eta\right|_{\eta=\eta_{G P}}}=\left.\int_{-1 / \sqrt{3}}^{1 / \sqrt{3}} \sqrt{\boldsymbol{X}_{, \eta} \cdot \boldsymbol{X}_{, \eta}}\right|_{\xi=\xi_{G P}} d \eta=\left.\frac{1}{\sqrt{3}} \sum_{\eta= \pm \frac{1}{3}} \sqrt{\boldsymbol{X}_{, \eta} \cdot \boldsymbol{X}_{, \eta}}\right|_{\xi=\xi_{G P}}
$$

\section{Numerical Examples}

To assess the performance of the present formulation, some problems have been

solved and the results are compared with available solutions in the literature or those obtained from simulations in the commercial FE software Ansys.

\subsection{Isotropic cantilever beam}

The shear-stress distribution through the thickness of an isotropic cantilever beam with rectangular cross section, subjected to an end transverse shear force, is studied and compared with the well-known parabolic expression:

$$
\tau_{x z}=\frac{V Q}{I b}=\frac{3 P}{2}\left(1-\frac{z^{2}}{c^{2}}\right)
$$

where $V, I$ and $Q$ are the shear force carried by the section, the second moment of area and the first moment of the area above distance of $\mathrm{z}$ about the neutral axis, respectively. The considered beam has the length of $L=10 \mathrm{~mm}$ and is under transverse end load of $P=1 \mathrm{MPa}$.

Fig 4 shows shear-stress values for different numbers of computational layers in the local coordinate system. In accordance with the schematic diagram Fig 2 , the shear stress is piecewise constant through the thickness of each layer and differences between the values at the interfaces decrease and the shear values at boundary layers become closer to boundary values with increasing number of computational layers. It can also be observed that the shear stress distribution, calculated via Eq (27), converges to the exact solution Eq (49) by increasing the number of layers.

\subsection{Laminated composite cantilever beam}

In this example the shear stress distribution through the thickness of a thick unidirectional (UD) as well as two cross-ply $[0 / 90]_{s}$ and $[90 / 0]_{s}$ composite cantilever beams with length-to-thickness ratio of 10 are studied [23]. The laminates 


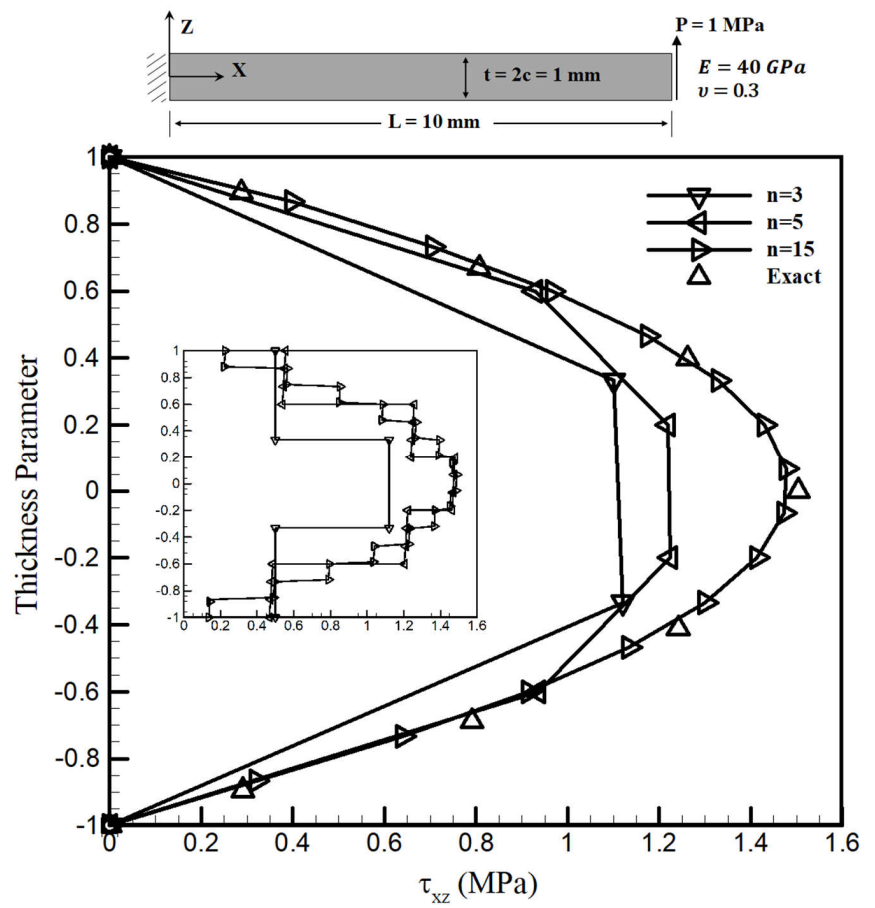

Figure 4: Interlaminar shear-stress distribution in an isotropic cantilever beam.

consist of equal-thickness layers of the ultra-high modulus orthotropic material GY70/Epoxy with material properties tabulated in Table 1.

Table 1: Material properties of GY70/Epoxy [23]

\begin{tabular}{|c|c|c|c|c|}
\hline$E_{1}(\mathrm{GPa})$ & $E_{2}(\mathrm{GPa})$ & $G_{12}(\mathrm{GPa})$ & $G_{23}(\mathrm{GPa})$ & $\nu_{12}$ \\
\hline 290 & 5 & 5 & 2.08 & 0.41 \\
\hline
\end{tabular}

As it can be observed in Fig 5, the obtained interlaminar shear-stress distributions compare very well with the results given in Ref [23]. It is worthy to note that the interlaminar shear-stress distribution in the $[0 / 90]_{s}$ laminate is similar to a trapezoid with the maximum value of $1.29 \mathrm{MPa}$ which is around 15 percent less than the maximum value of $1.5 \mathrm{MPa}$ in the parabolic distribution for the UD cantilever beam. Comparing the two cross-ply laminates, the maximum shear stress in the $[90 / 0]_{s}$ layup is more than twice of $[0 / 90]_{s}$ layup and has the value of 2.82 MPa. However, in this case interlaminar shear stress 
drops dramatically to very small values in $90^{\circ}$ face sheets which decreases the risk of delamination creation between 0 and 90 layers.

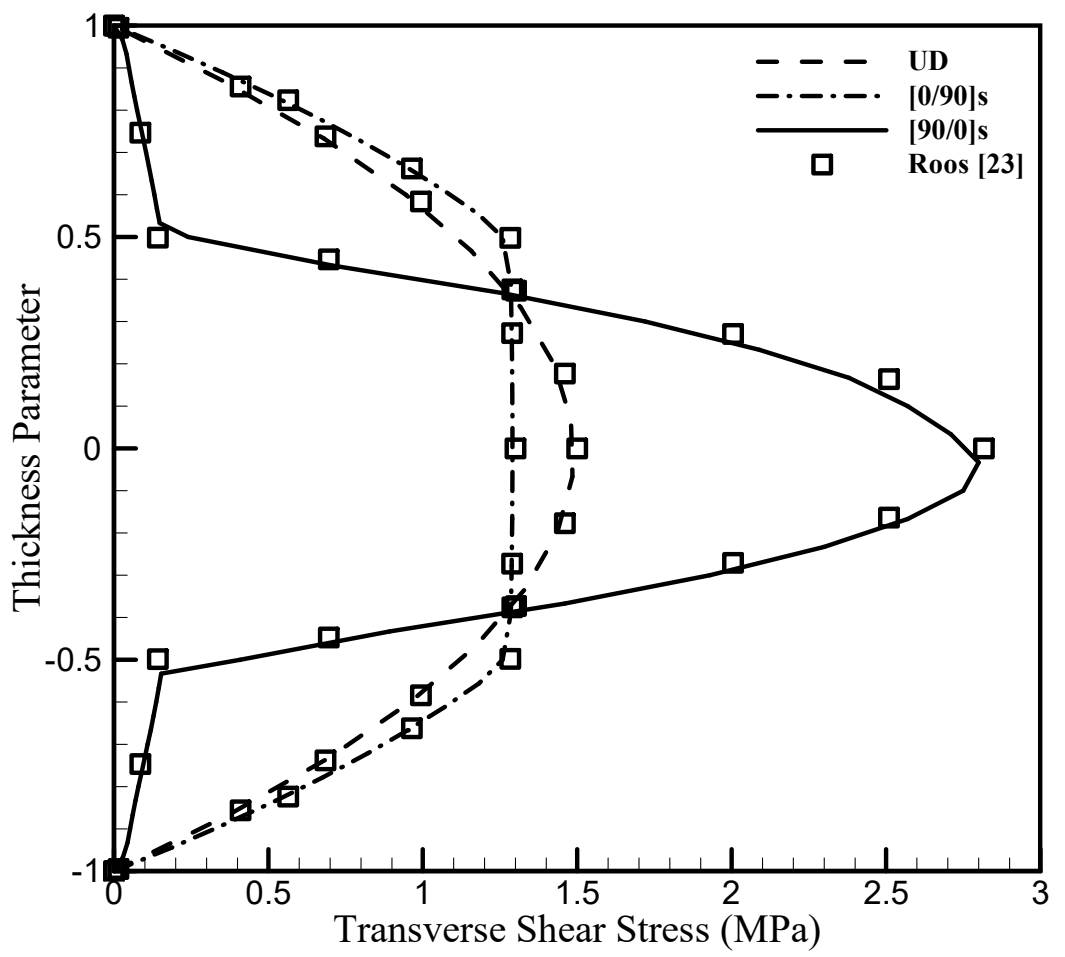

Figure 5: Interlaminar shear stress distribution in UD and cross-ply-laminate cantilever beams.

\subsection{Unsymmetric thick sandwich beam with highly heterogeneous material lay-} up

The distribution of shear stress is also studied through the thickness of an

unsymmetric typical sandwich construction: a highly compliant thick layer bounded by two relatively stiff and thin face sheets. The sandwich beam is relatively thick $\left(\frac{L}{t}=5\right)$ and its face sheets are highly anisotropic with the material properties given in Fig 6 . The soft inner and two stiff outer layers have thickness values of $t_{i}=16 \mathrm{~mm}$ and $t_{o}=2 \mathrm{~mm}$, respectively. 


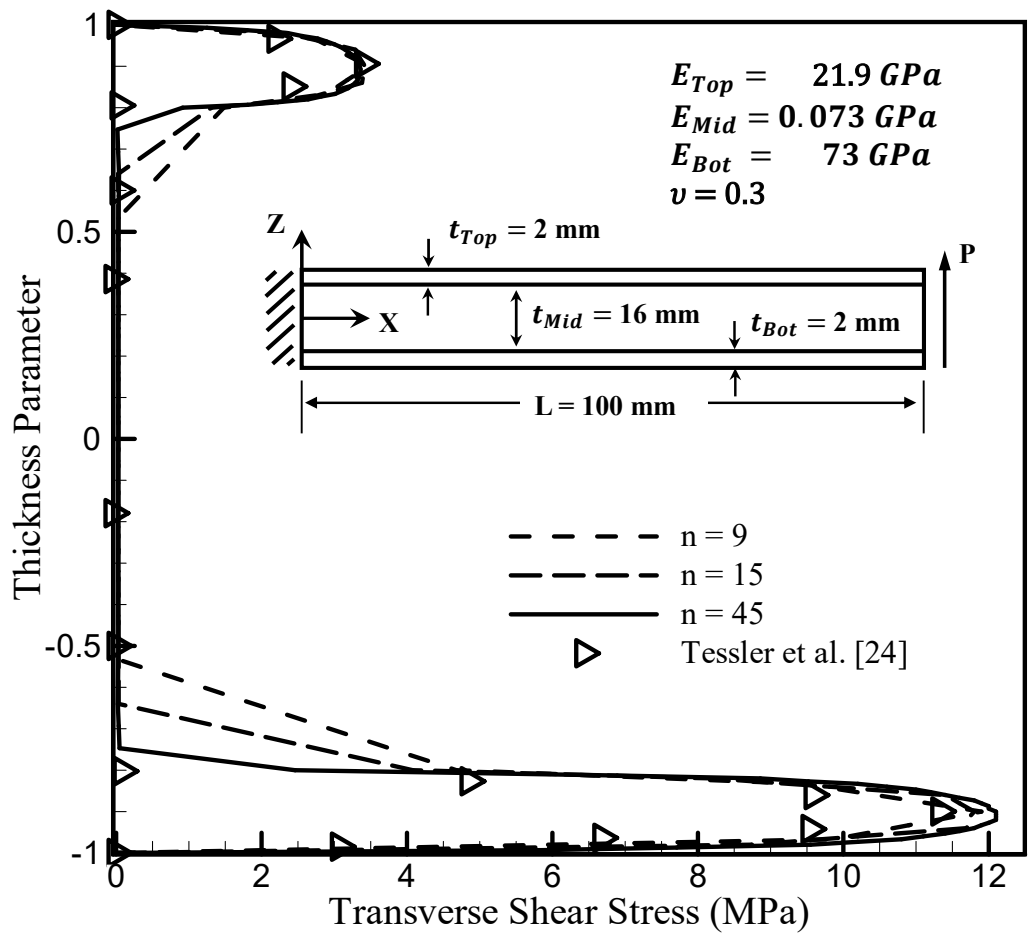

Figure 6: Interlaminar shear-stress distribution at the clamped end of an unsymmetrical thick sandwich beam with highly heterogeneous material lay-up.

Fig 6 shows the obtained shear stress distribution at the clamp end in comparison with results given in Ref [24]. As it can be observed, with increasing number of layers in the layer-wise shell element from $n=9$ to $n=45$, the significant change of interlaminar shear stress between the soft core and stiff face sheets can be captured very well. The results for the number of layers $n=45$ is in a good agreement with those presented in Ref [24].

\subsection{Curved sinusoidal composite shell}

This example deals with a sinusoidal composite shell [1] that the centreline follows the function $f$ : 


$$
f= \begin{cases}0 . & -100 \leq x<-50 \\ \frac{h_{\max }}{2}\left[1+\cos \left(\frac{2 \pi x}{L}\right)\right] & -50 \leq x \leq 50 \\ 0 . & 50<x \leq 100\end{cases}
$$

310

The material properties of composite are given in Fig 7.

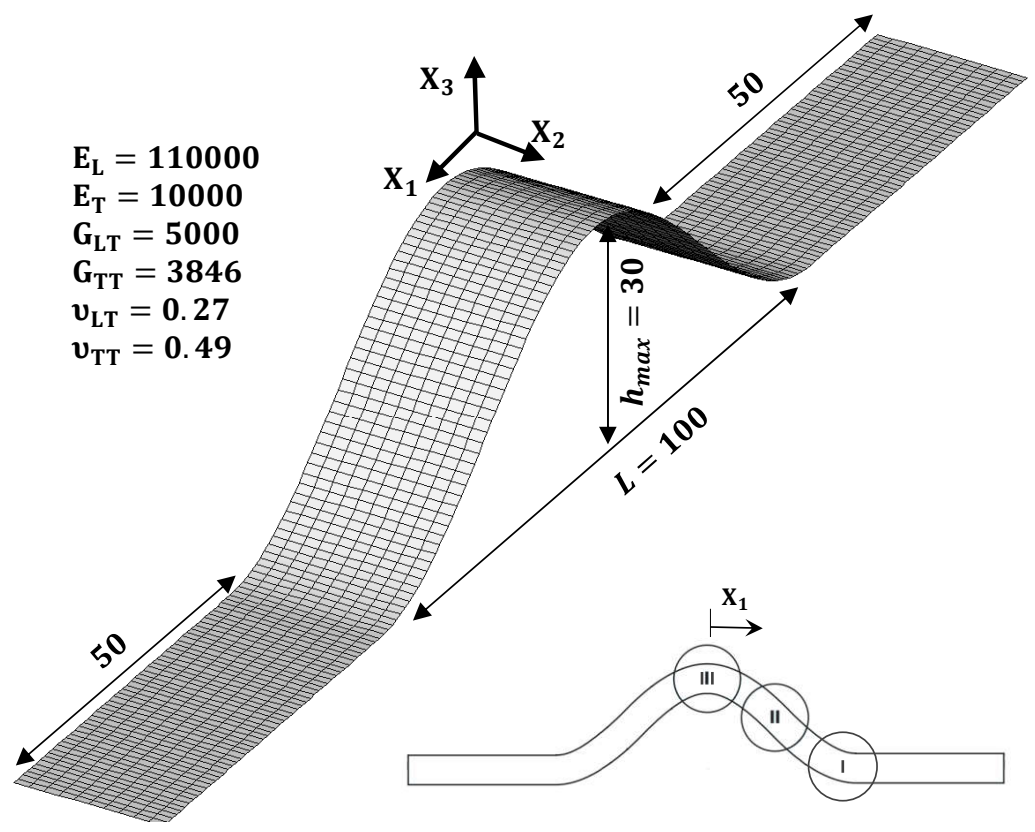

Figure 7: Mid-surface geometry of sinusoidal composite shell.

\subsubsection{Unidirectional sinusoidal composite shell}

Provoked by curvature effects, unbending deformation of specimens under tensile load results in high values of interlaminar normal stress at region III and partly region I in Fig 7. Fig 8(a) and (b) show interlaminar shear and normal stress distributions in a UD specimen under tensile load, obtained from geometrically nonlinear FE analysis in Ansys using the 3D element SOLID186 with 20 nodes. The specimen has a thickness of $5 \mathrm{~mm}$ and is modeled with a mesh with 300 elements along the sinusoidal curve and 15 elements along the thickness. 
The specimen is clamped at the left end and a line load with the magnitude of $F_{x}=125 \mathrm{~N} / \mathrm{mm}$ is applied at the right end, where the other degrees of freedom are suppressed.

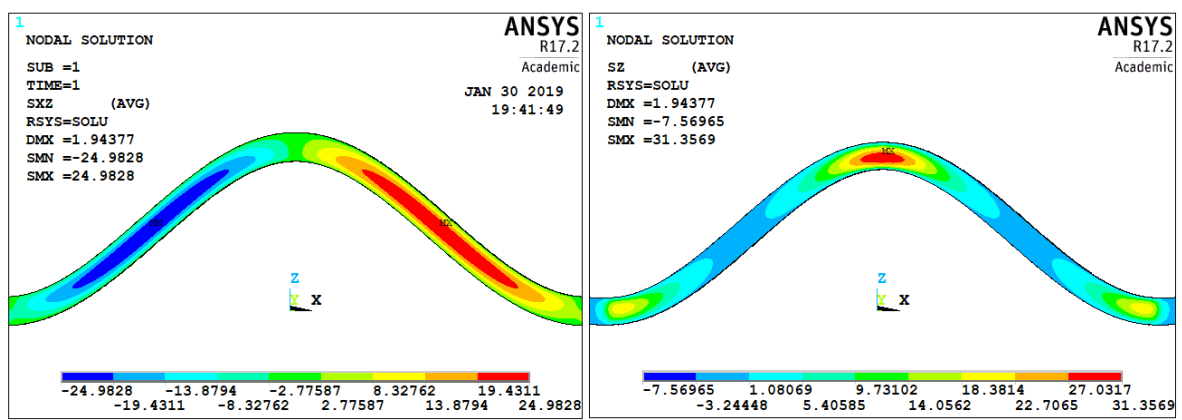

(a)

(b)

Figure 8: (a) Interlaminar shear-stress distribution (b) Interlaminar normal-stress distribution under tension loading in sinusoidal UD composite shell.

325

Using the layer-wise shell element for geometrically nonlinear analysis of a specimen under tension load, Fig 9 shows the interlaminar shear stress distributions along the thickness at region II, $x=25$. As it can be observed, by increasing the number of layers up to $n=9$, the obtained distribution converges to the results obtained by simulations with the commercial FE software [16]. 


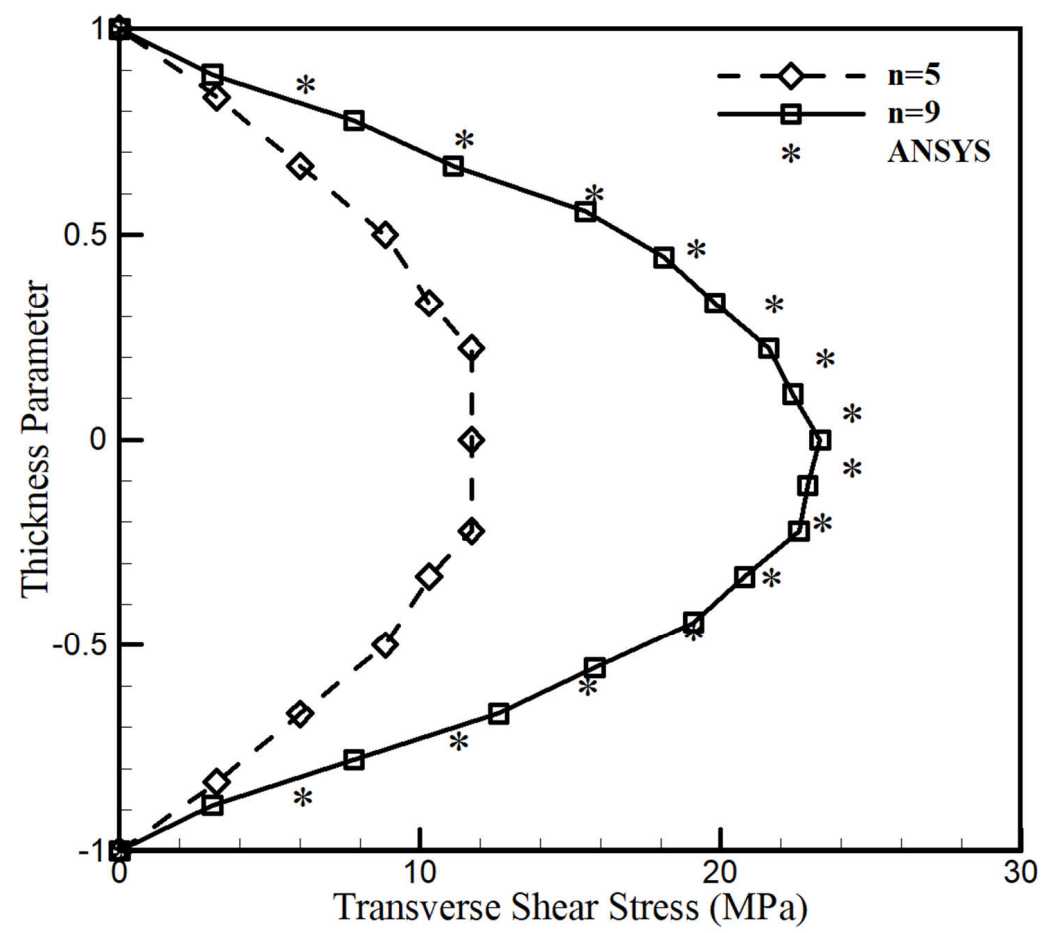

Figure 9: Interlaminar shear-stress distribution in sinusoidal UD composite shell at $\mathrm{x}=25$, within region II in Fig 7

Interlaminar normal-stress distribution along the thickness direction at region III, $x=0$, is calculated as it was discussed in section 3. Starting from zero boundary value on the bottom surface and using the backward finite-differencemethod (putting $A=1$ in Eq (44)), the interlaminar normal stress distribution is calculated and labeled as " $n=9$, Bottom BC" in Fig 10. It can be seen that, even though the obtained curve follows the parabolic distribution fairly well, it does not satisfy the boundary condition at the top surface. The distribution is also calculated based on the top boundary condition and using forward finitedifference-method (putting $A=-1$ in Eq (44)), and labeled as " $n=9$, Top BC" in Fig 10. Then the correct interlaminar normal stress distribution, which satisfies both boundary conditions, is determined using the linear Lagrange interpolation, Eq (45). 


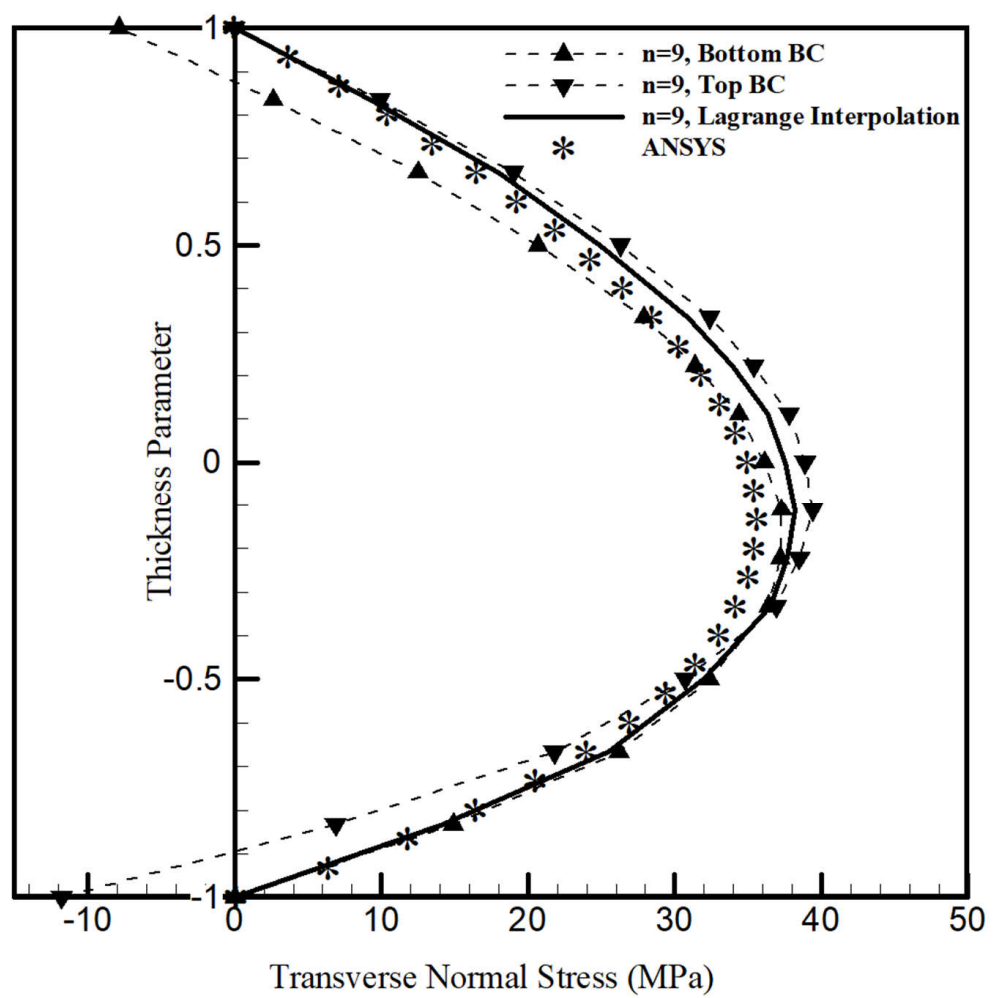

Figure 10: Interlaminar normal stress distribution in sinusoidal UD composite shell at $x=0$

Because of high values of interlaminar normal stress, the centre region III of the sinusoidal sample is prone for delamination onset. Relatively high values of interlaminar shear stress appeared in region II will drive the crack propagation[1].

\subsubsection{Cross-ply sinusoidal composite shells}

350

Interlaminar normal-stress distribution at the centre of sinusoidal composite shell is also calculated for two cross-ply lay-ups, $[0 / 90]_{s}$ and $[90 / 0]_{s}$. The crossply laminates have the same dimension given in Fig 7 and consist of equalthickness layers with total thickness of $5 \mathrm{~mm}$. The converged solutions are obtained using the layer-wise shell element with the number of layers $n=9$ and $355 \mathrm{n}=15$ for lay-ups of $[0 / 90]_{s}$ and $[90 / 0]_{s}$, respectively. The results are shown in Fig 11 and Fig 12. 


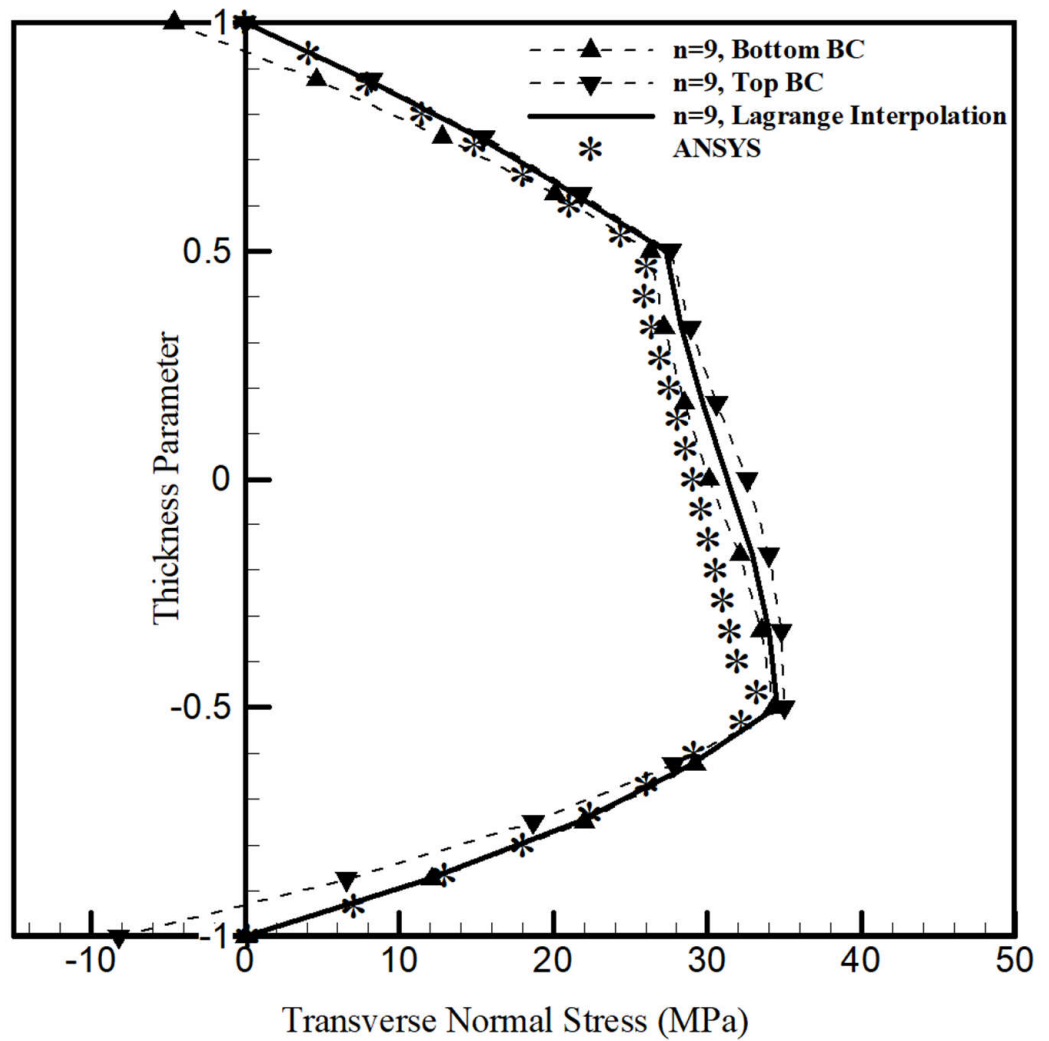

Figure 11: Interlaminar normal stress distribution in $[0 / 90]_{s}$ sinusoidal composite shell at $x=0$

It can be seen that the final distributions compare very well with the corresponding solid FE solutions. Comparing two cross-ply sinusoidal specimens, 360 interlaminar normal stress drops dramatically to very small values in $90^{\circ}$ face sheets for $[90,0]_{s}$ lay-up, which decreases the possibility of delamination creation between 0 and 90 layers. 


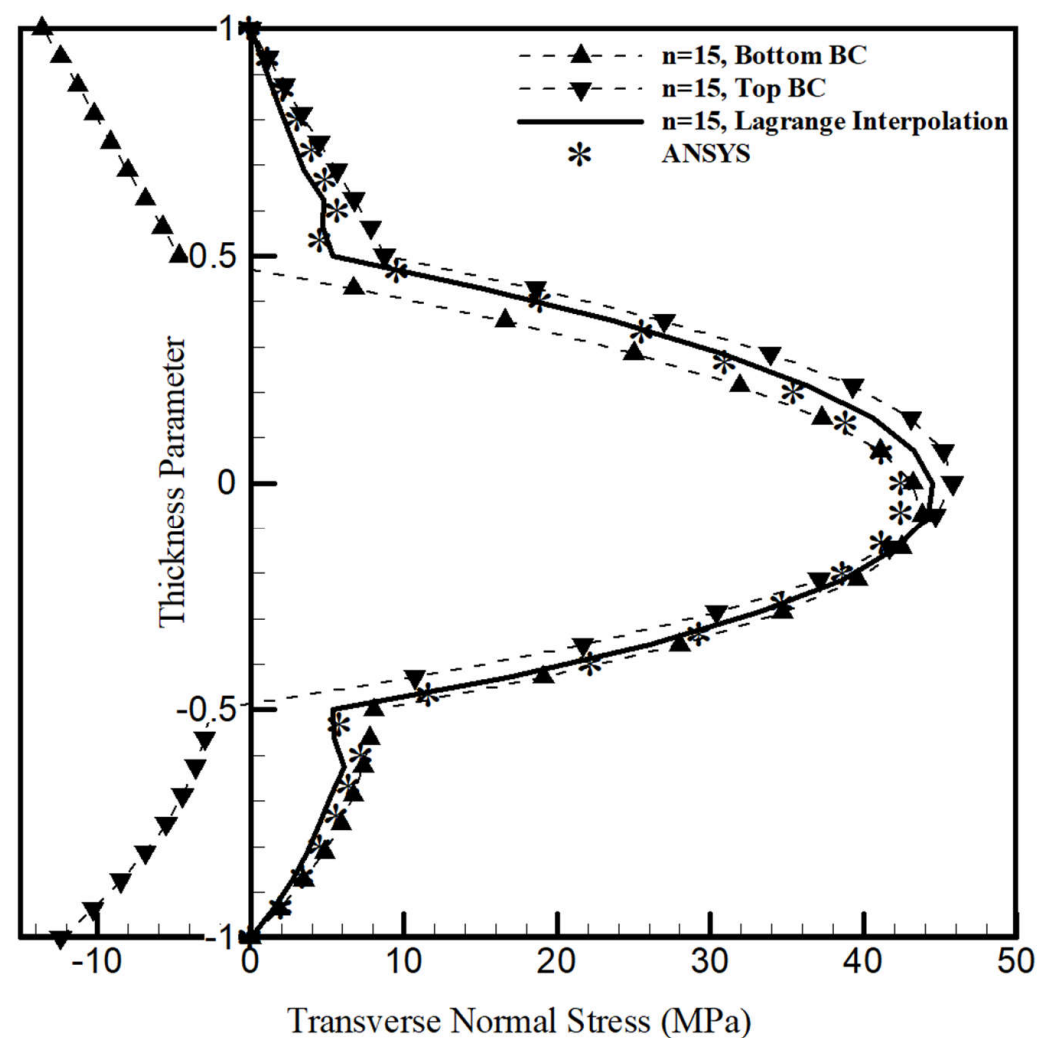

Figure 12: Interlaminar normal stress distribution in $[90 / 0]_{s}$ sinusoidal composite shell at $x=0$

Table 2 shows the significant improvement in the numerical size of FE model constructed by the layer-wise shell element with $n=15$ in comparison with the model consisting of the solid element.

Table 2: Numerical sizes of FE models by solid element and layer-wise shell element with number of layers $n=15$.

\begin{tabular}{|c|c|c|c|}
\cline { 2 - 4 } \multicolumn{1}{c|}{ FE model } & Node numbers & d.o.f per node & Total d.o.f \\
\hline Solid element & 182151 & 3 & 546453 \\
\hline Layer-wise shell element & 6021 & 33 & 198693 \\
\hline
\end{tabular}




\subsubsection{Free-edge effect in cross-ply laminate}

The final example studies free-edge stresses along 0/90 interface in a the vicinity of cross-ply $[0 / 90]_{s}$ composite laminate boundaries [25] [26] [27]. The laminate has width $L$ and thickness $h$, Fig 13 , with $\frac{L}{h}=10$ and is subjected to a constant longitudinal strain load $\epsilon_{y y}$ in width direction. Four material layers have the same thickness with elastic stiffness values shown in Fig 13.

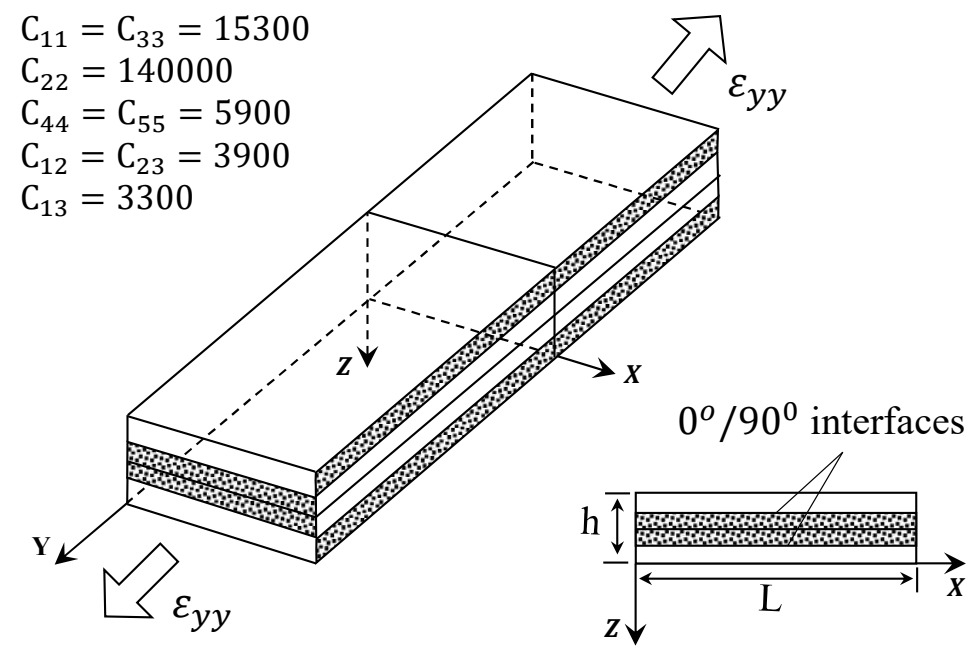

Figure 13: Cross-ply composite plate with free-edges under axial strain in width direction

Figure 14 compares the interlaminar shear and normal stress distribution obtained using the layer-wise shell element with $n=7$ along 0/90 interface with the published results. In accordance with literature results, interlaminar shear stress rises towards the free-edge but decreases rapidly to zero at $x=0$, dedicated by the traction-free edge condition. 


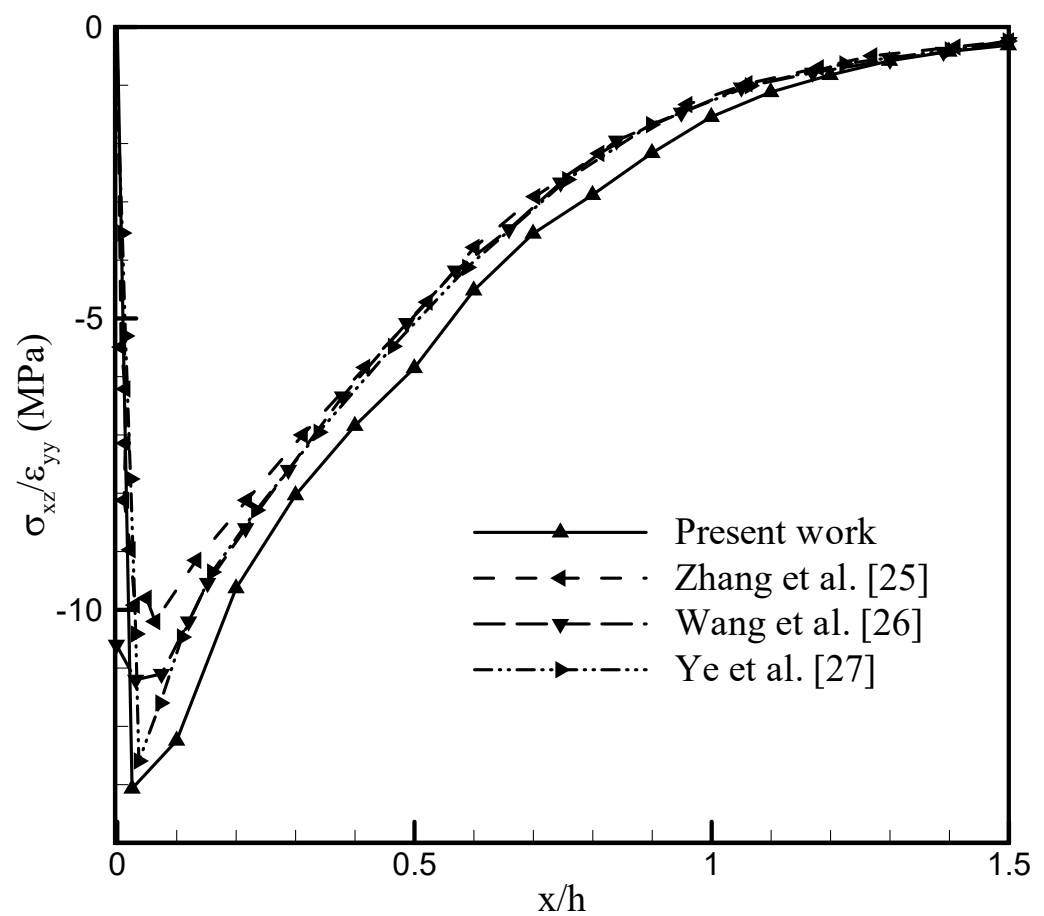

Figure 14: Distribution of interlaminar shear stress along $0^{\circ} / 90^{\circ}$ interface in $[0 / 90]_{s}$ laminate

Further, the distribution of the interlaminar normal stress near the free edge is calculated and compared in Fig 15 with those available in the literature. It is worth mentioning that the interlaminar stress disturbances occur only within a very local region near the boundaries, and approach zero towards the interior region, after $x>1.5 h$. 


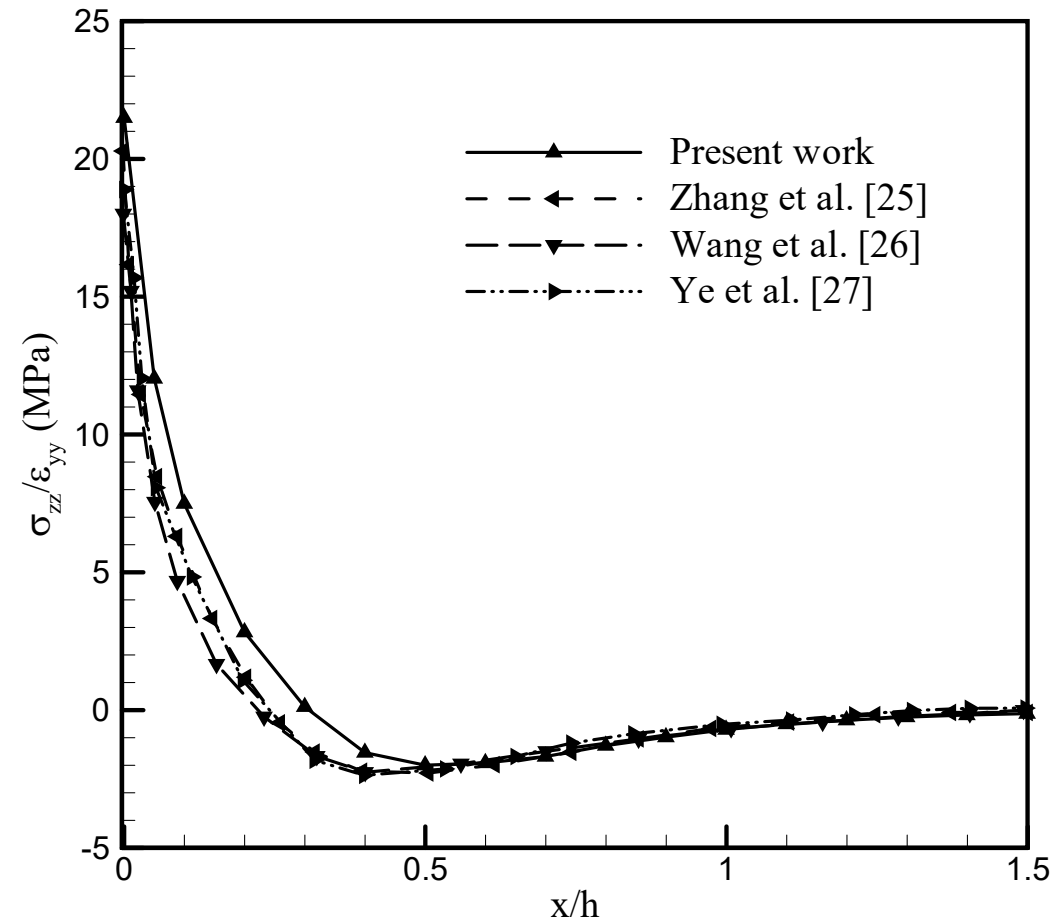

Figure 15: Distribution of interlaminar normal stress along $0^{\circ} / 90^{\circ}$ interface in $[0 / 90]_{s}$ laminate

\section{Conclusions}

A new scheme for calculating interlaminar stresses has been explained based on a novel geometrically nonlinear degenerated layer-wise shell finite element formulation incorporating zigzag shell theory. The interlaminar shear strains are kinematically coupled with the displacement solution so that they follow from the primary solution and it is only for interlaminar normal stresses that a post-processing step needs to be applied.

A proprietary FE code has been explained and the results for some sample problems have been compared with those available in the literature. For additional sample problems, comparison was made with simulations using the commercial FE software Ansys. The accuracy of the approximations depends on 
the number of mathematical layers used for modeling the laminates. It is shown that, with increasing number of those mathematical layers, the results converge quickly towards the respective true solutions at modest increase of numerical cost, in which adding each layer adds just two rotational degrees of freedom per nodes placed on the mid-surface.

The interlaminar normal stress distribution is then calculated through a post processing analysis and using the general form of the differential equation of equilibrium in a non-orthogonal element curvilinear coordinate system. Using the finite-difference-method in a curvilinear grid along the Gaussian points of element, the interlaminar normal stress is obtained by applying the bottom as well as the top surface boundary conditions to the 1st-order equation of equilibrium. To reflect the effect of two boundary conditions on the distribution, the linear Lagrange interpolation is applied to two solutions. The obtained result remarkably follows the correct distribution, in which the interlaminar normal stress at each point is calculated according the distance from each boundary. This applies even for the most complex distributions of interlaminar normal stresses that appear at the vicinity of free edge of multidirectional laminates.

\section{Acknowledgements}

The authors would like to acknowledge Dr. Gerald Kress at the Laboratory of Composite Material and Adaptive Structures, Department of Mechanical and Process Engineering, ETH Zurich, for sharing his expert knowledge with us and the comments that greatly improved the manuscript.

\section{Appendix}

$$
\begin{gathered}
{ }_{t}^{t+\Delta t} \boldsymbol{K}_{L \downarrow}^{l}=\frac{2 t_{k}^{l}}{t_{k}}\left({ }_{t}^{t+\Delta t} \boldsymbol{K}_{L}^{l(1)}-\frac{1}{2}{ }^{t+\Delta t} \boldsymbol{K}_{L}^{l(2)}+\frac{1}{3}{ }^{t+\Delta t} \boldsymbol{K}_{L}^{l(3)}\right) \\
{ }_{t}^{t+\Delta t} \boldsymbol{K}_{L}^{m}=\frac{2 t_{k}^{m}}{t_{k}}\left({ }_{t}^{t+\Delta t} \boldsymbol{K}_{L}^{m(1)}+\frac{1}{12}{ }^{t+\Delta t} \boldsymbol{K}_{L}^{m(3)}\right)
\end{gathered}
$$




$$
\begin{gathered}
{ }_{t}^{t+\Delta t} \boldsymbol{K}_{L \uparrow}^{l}=\frac{2 t_{k}^{l}}{t_{k}}\left({ }_{t}^{t+\Delta t} \boldsymbol{K}_{L}^{l(1)}+\frac{1}{2}{ }^{t+\Delta t} \boldsymbol{K}_{L}^{l(2)}+\frac{1}{3}{ }^{t+\Delta t} \boldsymbol{K}_{L}^{l(3)}\right) \\
{ }_{t}^{t+\Delta t} \boldsymbol{K}_{N L \downarrow}^{l}=\frac{2 t_{k}^{l}}{t_{k}}\left({ }_{t}^{t+\Delta t} \boldsymbol{K}_{N L}^{l(1)}-\frac{1}{2}{ }^{t+\Delta t} \boldsymbol{K}_{N L}^{l(2)}+\frac{1}{3}{ }^{t+\Delta t} \boldsymbol{K}_{N L}^{l(3)}-\frac{1}{4}{ }^{t+\Delta t} \boldsymbol{K}_{N L}^{l(4)}+\frac{1}{5}{ }^{t+\Delta t} \boldsymbol{K}_{N L}^{l(5)}\right) \\
{ }_{t}^{t+\Delta t} \boldsymbol{K}_{N L}^{m}=\frac{2 t_{k}^{l}}{t_{k}}\left({ }_{t}^{t+\Delta t} \boldsymbol{K}_{N L}^{m(1)}-\frac{1}{2}{ }^{t+\Delta t} \boldsymbol{K}_{N L}^{m(2)}+\frac{1}{3}{ }^{t+\Delta t} \boldsymbol{K}_{N L}^{m(5)}\right) \\
{ }_{t}^{t+\Delta t} \boldsymbol{K}_{N L \uparrow}^{l}=\frac{2 t_{k}^{l}}{t_{k}}\left({ }_{t}^{t+\Delta t} \boldsymbol{K}_{N L}^{l(1)}+\frac{1}{2}{ }^{t+\Delta t} \boldsymbol{K}_{N L}^{l(2)}+\frac{1}{3}{ }^{t+\Delta t} \boldsymbol{K}_{N L}^{l(3)}+\frac{1}{4}{ }^{t}{ }^{t+\Delta t} \boldsymbol{K}_{N L}^{l(4)}+\frac{1}{5}{ }^{t+\Delta t} \boldsymbol{K}_{N L}^{l(5)}\right) \\
{ }_{t}^{t+\Delta t} \boldsymbol{F}_{\downarrow}^{l}=\frac{2 t_{k}^{l}}{t_{k}}\left(_{t}^{t+\Delta t} \boldsymbol{F}^{l(1)}-\frac{1}{2}{ }^{t+\Delta t} \boldsymbol{F}^{l(2)}+\frac{1}{3}{ }^{t+\Delta t} \boldsymbol{F}^{l(3)}-\frac{1}{4}{ }^{t+\Delta t} \boldsymbol{F}^{l(4)}\right) \\
{ }_{t}^{t+\Delta t} \boldsymbol{F}^{m}=\frac{2 t_{k}^{l}}{t_{k}}\left(_{t}^{t+\Delta t} \boldsymbol{F}^{m(1)}+\frac{1}{12}{ }^{t+\Delta t} \boldsymbol{F}^{m(3)}\right) \\
{ }_{t}^{t+\Delta t} \boldsymbol{F}_{\uparrow}^{l}=\frac{2 t_{k}^{l}}{t_{k}}\left({ }_{t}^{t+\Delta t} \boldsymbol{F}^{l(1)}+\frac{1}{2}{ }^{t+\Delta t} \boldsymbol{F}^{l(2)}+\frac{1}{3}{ }^{t+\Delta t} \boldsymbol{F}^{l(3)}+\frac{1}{4}{ }^{t+\Delta t} \boldsymbol{F}^{l(4)}\right)
\end{gathered}
$$

${ }_{420}$ where

$$
\begin{aligned}
& { }_{t}^{t+\Delta t} \boldsymbol{K}_{L}^{l(1)}=\int_{-1}^{1} \int_{-1}^{1}{ }_{t}^{t+\Delta t} \boldsymbol{B}_{L a}^{l^{T}} \boldsymbol{C}^{l}{ }_{t}^{t+\Delta t} \boldsymbol{B}_{L a}^{l} d \xi d \eta \\
& { }_{t}^{t+\Delta t} \boldsymbol{K}_{L}^{l(2)}=\int_{-1}^{1} \int_{-1}^{1}\left({ }_{t}^{t+\Delta t} \boldsymbol{B}_{L a}^{l^{T}} \boldsymbol{C}^{l}{ }_{t}^{t+\Delta t} \boldsymbol{B}_{L b}^{l}+{ }_{t}^{t+\Delta t} \boldsymbol{B}_{L b t}^{l^{T}} \boldsymbol{C}_{t}^{l+\Delta t} \boldsymbol{B}_{L a}^{l}\right) d \xi d \eta \\
& { }_{t}^{t+\Delta t} \boldsymbol{K}_{L}^{l(3)}=\int_{-1}^{1} \int_{-1}^{1}{ }_{t}^{t+\Delta t} \boldsymbol{B}_{L b t}^{l^{T}} \boldsymbol{C}_{t}^{l+t+\Delta t} \boldsymbol{B}_{L b}^{l} d \xi d \eta \\
& { }_{t}^{t+\Delta t} \boldsymbol{K}_{N L}^{l(1)}=\int_{-1}^{1} \int_{-1}^{1}{ }^{t+\Delta t} \boldsymbol{B}_{N L a}^{l^{T}}{ }^{t+\Delta t} \boldsymbol{S}^{l(0)^{*}}{ }_{t}^{t+\Delta t} \boldsymbol{B}_{N L a}^{l} d \xi d \eta \\
& { }_{t}^{t+\Delta t} \boldsymbol{K}_{N L}^{l(2)}=\int_{-1}^{1} \int_{-1}^{1}\left({ }_{t}^{t+\Delta t} \boldsymbol{B}_{N L a t}^{l^{T}}{ }^{t+\Delta t} \boldsymbol{S}^{l(0)^{*}}{ }_{t}^{t+\Delta t} \boldsymbol{B}_{N L b}^{l}\right. \\
& \left.+{ }_{t}^{t+\Delta t} \boldsymbol{B}_{N L a t}^{l^{T}}{ }^{t+\Delta t} \boldsymbol{S}^{l(1)^{*}}{ }_{t}^{t+\Delta t} \boldsymbol{B}_{N L a}^{l}+{ }_{t}^{t+\Delta t} \boldsymbol{B}_{N L b t}^{l^{T}}{ }^{t+\Delta t} \boldsymbol{S}^{l(2)^{*}}{ }_{t}^{t+\Delta t} \boldsymbol{B}_{N L a}^{l}\right) d \xi d \eta
\end{aligned}
$$




$$
\begin{aligned}
& { }_{t}^{t+\Delta t} \boldsymbol{K}_{N L}^{l(3)}=\int_{-1}^{1} \int_{-1}^{1}\left({ }_{t}^{t+\Delta t} \boldsymbol{B}_{N L a t}^{l^{T}}{ }^{t+\Delta t} \boldsymbol{S}^{l(2)^{*}}{ }_{t}^{t+\Delta t} \boldsymbol{B}_{N L a}^{l}\right. \\
& +{ }_{t}^{t+\Delta t} \boldsymbol{B}_{N L a}^{l^{T}{ }^{t+\Delta t}}{ }^{t+\Delta(1)^{*}}{ }_{t}^{t+\Delta t} \boldsymbol{B}_{N L b}^{l}+{ }_{t}^{t+\Delta t} \boldsymbol{B}_{N L b t}^{l^{T}}{ }^{t+\Delta t} \boldsymbol{S}^{l(1)^{*}}{ }_{t}^{t+\Delta t} \boldsymbol{B}_{N L a}^{l} \\
& \left.+{ }_{t}^{t+\Delta t} \boldsymbol{B}_{N L b t}^{l^{T}}{ }^{t+\Delta t} \boldsymbol{S}^{l(0)^{*}}{ }_{t}^{t+\Delta t} \boldsymbol{B}_{N L b}^{l}\right) d \xi d \eta \\
& { }_{t}^{t+\Delta t} \boldsymbol{K}_{N L}^{l(4)}=\int_{-1}^{1} \int_{-1}^{1}\left({ }_{t}^{t+\Delta t} \boldsymbol{B}_{N L a t}^{l^{T}}{ }^{t+\Delta t} \boldsymbol{S}^{l(2)^{*}}{ }_{t}^{t+\Delta t} \boldsymbol{B}_{N L b}^{l}\right. \\
& \left.+{ }_{t}^{t+\Delta t} \boldsymbol{B}_{N L b t}^{l^{T}}{ }^{t+\Delta t} \boldsymbol{S}^{l(2)^{*}}{ }_{t}^{t+\Delta t} \boldsymbol{B}_{N L a}^{l}+{ }_{t}^{t+\Delta t} \boldsymbol{B}_{N L b t}^{l^{T}}{ }^{t+\Delta t} \boldsymbol{S}^{l(1)^{*}}{ }_{t}^{t+\Delta t} \boldsymbol{B}_{N L a}^{l}\right) d \xi d \eta \\
& { }_{t}^{t+\Delta t} \boldsymbol{K}_{N L}^{l(5)}=\int_{-1}^{1} \int_{-1}^{1}{ }_{t}^{t+\Delta t} \boldsymbol{B}_{N L b t}^{l^{T}}{ }^{t+\Delta t} \boldsymbol{S}^{l(2)^{*}}{ }_{t}^{t+\Delta t} \boldsymbol{B}_{N L b}^{l} d \xi d \eta \\
& { }_{t}^{t+\Delta t} \boldsymbol{F}^{(1)}=\int_{-1}^{1} \int_{-1}^{1}{ }^{t+\Delta t} \boldsymbol{B}_{L a}^{l^{T}}{ }^{t} \hat{\boldsymbol{\sigma}}^{l(0)} d \xi d \eta \\
& { }_{t}^{t+\Delta t} \boldsymbol{F}^{(2)}=\int_{-1}^{1} \int_{-1}^{1}\left({ }_{t}^{t+\Delta t} \boldsymbol{B}_{L b}^{l^{T}}{ }^{t} \hat{\boldsymbol{\sigma}}^{l(0)}+{ }_{t}^{t+\Delta t} \boldsymbol{B}_{L a}^{l^{T}}{ }^{t} \hat{\boldsymbol{S}}^{l(1)}\right) d \xi d \eta \\
& { }_{t}^{t+\Delta t}{ }^{(3)}=\int_{-1}^{1} \int_{-1}^{1}\left({ }_{t}^{t+\Delta t}{ }^{(+\Delta} \boldsymbol{B}_{L a}^{l^{T}}{ }^{t} \hat{\boldsymbol{S}}^{l(2)}+{ }_{t}^{t+\Delta t}{ }^{t+} \boldsymbol{B}_{L b}^{l^{T}}{ }^{t} \hat{\boldsymbol{S}}^{l(1)}\right) d \xi d \eta \\
& { }_{t}^{t+\Delta t} \boldsymbol{F}^{(4)}=\int_{-1}^{1} \int_{-1}^{1}{ }^{t+\Delta t}{ }^{{ }^{2}}{ }_{L b}^{l^{T}}{ }^{t} \hat{\boldsymbol{S}}^{l(2)} d \xi d \eta
\end{aligned}
$$

More details on derivation of the layer-wise shell element formulation can be found in Refs [28] and [29].

\section{References}

[1] R. Roos, G. Kress, M. Barbezat, P. Ermanni, Enhanced model for interlaminar normal stress in singly curved laminates, Composite Structures 80 (3) (2007) 327-333.

[2] R. Tanov, A. Tabiei, Adding transverse normal stresses to layered shell finite elements for the analysis of composite structures, Composite structures 76 (4) (2006) 338-344. 
[3] G. Kulikov, S. Plotnikova, Non-linear strain-displacement equations exactly representing large rigid-body motions. part ii. enhanced finite element technique, Computer Methods in Applied Mechanics and Engineering 195 (19-22) (2006) 2209-2230.

[4] D. Marinković, G. Rama, M. Zehn, Abaqus implementation of a corotational piezoelectric 3-node shell element with drilling degree of freedom, Facta Universitatis, Series: Mechanical Engineering 17 (2) (2019) 269-283.

[5] K. Rah, W. Van Paepegem, A. Habraken, J. Degrieck, A mixed solid-shell element for the analysis of laminated composites, International Journal for numerical methods in engineering 89 (7) (2012) 805-828.

${ }_{440}$ [6] J. González-Cantero, E. Graciani, F. París, B. López-Romano, Semianalytic model to evaluate non-regularized stresses causing unfolding failure in composites, Composite Structures 171 (2017) 77-91.

[7] A. Nagle, D. Wowk, C. Marsden, Three-dimensional modelling of interlaminar normal stresses in curved laminate components, Composite Structures (2020) 112165.

[8] C. Thurnherr, L. Ruppen, G. Kress, P. Ermanni, Interlaminar stresses in corrugated laminates, Composite Structures 140 (2016) 296-308.

[9] D. Filipovic, G. R. Kress, Stress analysis of corrugated orthotropic laminates under transverse shear loading, Composite Structures 223 (2019) 110983.

[10] A. Solis, S. Sanchez-Saez, X. Martinez, E. Barbero-Pozuelo, Numerical analysis of interlaminar stresses in open-hole laminates under compression, Composite Structures 217 (2019) 89-99.

[11] G. Kulikov, E. Carrera, Finite deformation higher-order shell models and 455 rigid-body motions, International Journal of Solids and Structures 45 (1112) (2008) 3153-3172. 
[12] E. Carrera, S. Valvano, M. Filippi, Classical, higher-order, zig-zag and variable kinematic shell elements for the analysis of composite multilayered structures, European Journal of Mechanics-A/Solids 72 (2018) 97-110.

[13] S. Valvano, E. Carrera, Multilayered plate elements with node-dependent kinematics for the analysis of composite and sandwich structures, Facta Universitatis, Series: Mechanical Engineering 15 (1) (2017) 1-30.

[14] M. Filippi, E. Carrera, S. Valvano, Analysis of multilayered structures embedding viscoelastic layers by higher-order, and zig-zag plate elements, Composites Part B: Engineering 154 (2018) 77-89.

[15] A. Pagani, S. Valvano, E. Carrera, Analysis of laminated composites and sandwich structures by variable-kinematic mitc9 plate elements, Journal of Sandwich Structures \& Materials 20 (1) (2018) 4-41.

[16] S.-N. Nguyen, J. Lee, M. Cho, Viscoelastic behavior of naghdi shell model based on efficient higher-order zig-zag theory, Composite Structures 164 (2017) 304-315.

[17] S. K. Kumar, D. Harursampath, E. Carrera, M. Cinefra, S. Valvano, Modal analysis of delaminated plates and shells using carrera unified formulationmitc9 shell element, Mechanics of Advanced Materials and Structures 25 (8) (2018) 681-697.

[18] E. Carrera, G. Giunta, M. Petrolo, Beam structures: classical and advanced theories, John Wiley \& Sons, 2011.

[19] M. Hajikazemi, W. Van Paepegem, Variational analysis of free-edge stress and displacement fields in general un-symmetric and thin-ply laminates under in-plane, bending and thermal loading, Composites Part A: Applied Science and Manufacturing 113 (2018) 220-232.

[20] D. Wowk, C. Marsden, D. Thibaudeau, Predicting the relative magnitude of interlaminar stresses due to edge effects in thin angle-ply laminates using macroscopic finite element modeling, Composite Structures (2020) 112164. 
[21] J. Argyris, An excursion into large rotations, Computer methods in applied mechanics and engineering 32 (1-3) (1982) 85-155.

[22] K. J. Bathe, Finite element procedures, Prentice-Hall, Upper Saddle River, New Jersy, 1996.

[23] R. Roos, Model for interlaminar normal stresses in doubly curved laminates,

[24] A. Tessler, M. Di Sciuva, M. Gherlone, A refined zigzag beam theory for composite and sandwich beams, Journal of Composite Materials 43 (9) (2009) 1051-1081.

[25] D. Zhang, J. Ye, H. Y. Sheng, Free-edge and ply cracking effect in cross-

[29] Z. Soltani, S. H. Kordkheili, G. Kress, Experimental and numerical study of geometrically nonlinear behavior of corrugated laminated composite shells using a nonlinear layer-wise shell fe formulation, Engineering Structures 184 (2019) 61-73. 